\title{
An assessment of scale-dependent variability and bias in global prediction models
}

\author{
Nedjeljka Žagar ${ }^{1,2}$ (1) $\cdot$ Katarina Kosovelj $^{3} \cdot$ Elisa Manzini $^{4} \cdot$ Martin Horvat $^{3} \cdot$ José Castanheira $^{5}$
}

Received: 6 April 2018 / Accepted: 25 September 2019 / Published online: 8 October 2019

\begin{abstract}
The paper presents a method for the scale-dependent validation of the spatio-temporal variability in global weather or climate models and for their bias quantification in relation to dynamics. The method provides a relationship between the bias and simulated spatial and temporal variance by a model in comparison with verifying reanalysis data. For the low resolution (T30L8) subset of ERA-20C data, it was found that 80-90\% (depending on season) of the global interannual variance is at planetary scales (zonal wavenumbers $k=0-3$ ), and only about $1 \%$ of the variance is at scales with $k>7$. The reanalysis is used to validate a T30L8 GCM in two configurations, one with the prescribed sea-surface temperature (SST) and another using a slab ocean model. Although the model with the prescribed SST represents the average properties of surface fields well, the interannual variability is underestimated at all scales. Similar to variability, model bias is strongly scale dependent. Biases found in the experiment with the prescribed SST are largely increased in the experiment using a slab ocean, especially in $k=0$, in scales with missing variability and in seasons with poorly simulated energy distribution. The perfect model scenario (a comparison between the GCM coupled to a slab ocean vs. the same model with prescribed SSTs) shows that the representation of the ocean is not critical for synoptic to subsynoptic variability, but essential for capturing the planetary scales.
\end{abstract}

Keywords Spatio-temporal variability $\cdot$ Bias spectra $\cdot$ Variability quantification $\cdot$ Climate models $\cdot$ Model validation

Nedjeljka Žagar

nedjeljka.zagar@uni-hamburg.de

Katarina Kosovelj

katarina.kosovelj@fmf.uni-lj.si

Elisa Manzini

elisa.manzini@mpimet.mpg.de

Martin Horvat

martin.horvat@fmf.uni-lj.si

José Castanheira

jcast@ua.pt

1 Present Address: Meteorologisches Institut, CEN, Universität Hamburg, Grindelberg 7, 20144 Hamburg, Germany

2 Faculty of Mathematics and Physics, University of Ljubljana, Jadranska 19, 1000 Ljubljana, Slovenia

3 University of Ljubljana, Jadranska 19, 1000 Ljubljana, Slovenia

4 Max-Planck-Institut fur Meteorologie, Bundesstraße 53, 20146 Hamburg, Germany

5 CESAM and Department of Physics, University of Aveiro, Campus de Santiago, 3810-193 Aveiro, Portugal

\section{Introduction}

Atmospheric general circulation models (GCMs) are frequently validated with the reanalysis data to test their reliability to reproduce the observed mean state and variability. Although reanalyses are a mixture of observations and model information and as such contain uncertainties, they are the most representative picture of the present climate. Before using a GCM for climate projections, one first tests whether it can reproduce the global properties of circulation (winds) and thermodynamical variables (temperature, pressure and moisture fields) in agreement with reanalyses, based on the observed forcing parameters (e.g., greenhouse gas concentration).

The two most relevant parameters of the surface climate are 2-m temperature and precipitation which can be validated with long observation records. In contrast, circulation is difficult to validate (Shepherd 2014) and the results may be inconclusive (Knutti and Sedlacek 2013). The comparison of zonal mean values of wind, temperature and moisture with zonally-averaged reanalysis data quantifies how well 
the GCMs represent the zonal wavenumber zero (e.g Flato et al. 2013). A quantitative validation of simulated wave variance is more difficult to perform, especially as it is controlled by the zonal mean circulation.

Simulated zonal mean flows also greatly affect teleconnections (e.g. Branstator 2002). Forcing for teleconnections is provided by subseasonal variability; its reliable simulation is a testing ground for seamless prediction (Palmer et al. 2008). A basis for seamless prediction are the medium-range numerical weather prediction (NWP) models that are first adapted for subseasonal and seasonal forecasting systems (e.g. Vitart et al. 2008), then extended to climate time scales (e.g. EC-Earth, http://www.ec-earth.org).

NWP models are frequently validated by using their energy spectra (e.g. Wedi 2014). In a global atmosphere characterized by energy and enstrophy conservation, timeaveraged energy spectra are expected to have wavenumber ${ }^{-3}$ dependence for a range of synoptic scales, and a shallower slope for planetary scales as well as for subsynoptic scales (Hoskins and James 2014, Chapter 11). On synoptic scales, the energy spectrum encompasses the quasi-geostrophic dynamics in terms of the approximately non-divergent Rossby waves. On subsynoptic scales, shallowing of the spectrum from -3 towards $-5 / 3$ in NWP models appears associated with the divergence-dominated, inertio-gravity wave oscillations (e.g. Žagar et al. 2017). The departure from the expected spectrum is one measure of unrealistic spatial variability in the model. A typical feature of NWP models is a lack of variability at small scales commonly associated with insufficient representation of physical processes and numerical filtering (e.g. Blažica et al. 2013).

This paper extends the scale-dependent analysis of global variability from the spatial to the temporal domain. We define scale-dependent measures of temporal variability and model bias and apply them for the validation of a global climate model using reanalysis data.

The scale-dependent validation of climate model variability is a challenging topic because there is no single definition of variability. Under the global constraints of mass, energy and momentum, GCMs simulate the spectrum of variability from weather to decadal time scales across many spatial scales. Temporal variability can be split among various time scales, variables and regions whereas details of the spatial spectrum associated with certain temporal frequency can be described as a combination of "preferred" patterns.

The most prominent patterns of climate variability have been identified through spatial teleconnections defined by correlations between regional climate variations at widely separated, geographically fixed spatial locations and by principal component analysis (Cubasch et al. 2013). Equatorial variability in GCMs has been validated by comparing the space-time filtered fields in reanalyses and models (e.g. Lin et al. 2006; Hung et al. 2013) whereas midlatitude variability has been evaluated by comparing indices such as the NorthAtlantic oscillation index, the Arctic oscillation index and the blocking index (e.g. Scaife et al. 2012). Validation of planetary waves is relevant both for the tropics and the midlatitudes; for instance, they are key to the dynamical coupling between the troposphere and stratosphere. Stratospheric variability, and its impact on the troposphere, is poorly simulated in many climate models (e.g. Charlton-Perez et al. 2013).

As for circulation, teleconnections exist also among the patterns of bias in the models. For example, Wang et al. (2014) described links between biases in distant regions of atmosphere and oceans in 22 climate models. They show that efforts for improving simulations of regional patterns of variability can be overridden by remote biases in the models. This provides a strong motivation for a simultaneous validation of the amplitude and phase of the bias and spatiotemporal variability in the models.

We introduce a scale-dependent validation of global models using the projection of the atmospheric state into a set of three-dimensionally orthogonal modes that provide physical basis for the interpretation of atmospheric circulation. The proposed method is envisaged for the comparison of models against reanalyses and for the intercomparison of the global multi-model ensembles, such as the Coupled Model Intercomparison Project (CMIP) ensembles (Eyring et al. 2016).

As an example, the method is here applied to the recent ECMWF reanalysis dataset-ERA-20C (Poli et al. 2016) to quantify the observed (i.e. reanalysis) variability spectrum. The observed spectrum is then compared to the spectrum of a low-resolution GCM and the modal decomposition is also used to examine the spectrum of the model bias.

The presented application utilizes monthly mean time series from the reanalysis and model simulations but there is no restriction to the inclusion of higher/lower frequencies in the considered time series. Furthermore, the decomposition differentiates between the circulation which projects onto quasi-geostrophic, approximately nondivergent dynamics represented by the Rossby waves and the predominantly irrorational dynamics represented by the inertio-gravity modes.

Among the variety of questions that can be addressed by the proposed method, we ask the following questions:

- What is the amplitude of the global circulation variability and how does it vary with season? What percentage of the global variability in reanalysis data is simulated by GCMs when forced by the observed sea-surface temperature (SST)?

- How is the global circulation variability distributed as a function of zonal scale? For example, what percentage of variability is in the zonal mean state and how much variability is left beyond the planetary scale (i.e. for zonal wavenumber larger than 3 )? 
- How does the amplitude of circulation variability in a GCM compare to its bias at different spatial scales?

We attempt to discuss bias and temporal variability in relation to the spatial variability (energy spectra) in a GCM. We focus on scales larger than $1000 \mathrm{~km}$ in hydrostatic atmosphere. With the zonal scale $L$ defined as $L=a \cos (\phi) \pi / k$, where $k$ is the zonal wavenumber index, $\phi$ is latitude and $a=6371 \mathrm{~km}$ is the average Earth radius, $L=1000 \mathrm{~km}$ scale corresponds to zonal wavenumbers 0-20 in the tropics, and to wavenumbers 0-14 in the midlatitudes.

Global validation of GCM variability and biases and their reduction will diminish the need for climate model bias removal in order to use the surface outputs for climate change impact studies (e.g. Ehret et al. 2012). Small improvements in simulated global circulation patterns can bring about significantly larger improvements in regional climate models which are forced by these fields.

The outline of the paper is the following. In the next section we describe how to calculate the scale-dependent global variability and bias of the atmospheric state by projecting the atmospheric state into a set of three-dimensionally orthogonal modes, using the normal-mode function decomposition. Section 3 introduces the setup of numerical simulations, reanalysis data and the specific of the scale decomposition for the current comparison. Section 4 presents the spectra of variability and energy in the model and reanalysis data and the bias spectra. Discussion of biases in physical space is given in Sect. 5. Section 6 contains conclusions and outlook.

\section{Formulation of the scale-dependent validation of GCMs using reanalysis data}

We start by reviewing the derivation of scale-dependent decomposition of three-dimensional atmospheric geopotential and wind fields in horizontal and vertical scales using the normal-mode functions. Then we introduce the three key quantities for the model validation: spatial variability (energy spectra), temporal variability (variance spectra) and bias (model versus reanalysis data).

\subsection{Scale decomposition using the normal-mode functions}

The normal-mode function (NMF) are analytical solutions of the linearized equations describing oscillations of the zonal wind $\left(u^{\prime}\right)$, meridional wind $\left(v^{\prime}\right)$ and geopotential height $\left(h^{\prime}\right)$, superimposed on a basic state with temperature $T_{0}$ (e.g. Daley $1991)$. For the system with the vertical $\sigma$ coordinate, the linearized adiabatic equations for oscillations $\left(u^{\prime}, v^{\prime}, h^{\prime}\right)$ around the basic state of rest were derived by Kasahara and Puri (1981) as: $\frac{\partial u^{\prime}}{\partial t}-2 \Omega v^{\prime} \sin (\varphi)=-\frac{g}{a \cos (\varphi)} \frac{\partial h^{\prime}}{\partial \lambda}$,

$\frac{\partial v^{\prime}}{\partial t}+2 \Omega u^{\prime} \sin (\varphi)=-\frac{g}{a} \frac{\partial h^{\prime}}{\partial \varphi}$,

$\frac{\partial}{\partial t}\left[\frac{\partial}{\partial \sigma}\left(\frac{g \sigma}{R \Gamma_{0}} \frac{\partial h^{\prime}}{\partial \sigma}\right)\right]-\nabla \cdot \mathbf{V}^{\prime}=0$.

The parameter $a$ denotes the Earth's radius, $\Omega$ is the Earth's rotation rate, $R$ is the gas constant of air and $g$ is the Earth's gravity. Equation (3) is obtained as a combination of the continuity and thermodynamic equations, using the pseudo geopotential height variable, $h=P / g$, where $P=g z+R T_{0} \ln \left(p_{s}\right)$, with $z$ denoting the hydrostatic height of a terrain-following $\sigma$ level, $\sigma=\left(p-p_{T}\right) /\left(p_{s}-p_{T}\right)$. Here, $p_{T}$ is the top level pressure $\left(p_{T}=0\right)$ and $p_{s}$ is the surface pressure. The pseudo geopotential height anomaly $h^{\prime}$ is defined as $h^{\prime}=P^{\prime} / g$, where $P^{\prime}$ is the anomaly of $P$ relative to the globally averaged $P$ at each $\sigma$ level. The static stability parameter $\Gamma_{0}$ is defined as $\Gamma_{0}=\kappa T_{0} / \sigma-\mathrm{d} T_{0} / \mathrm{d} \sigma$, where $\kappa=R / c_{p}, c_{p}$ is the specific heat of air at constant pressure and $T_{0}$ is the globally averaged temperature at a $\sigma$ level. The boundary conditions for the system of Eqs. (1)-(3) are derived from the assumption that there is no mass transport across the surfaces $\sigma=0$ and $\sigma=1$ :

$\dot{\sigma}=0$ at the surface, $\sigma=1$, and at the top, $\sigma=0$.

For details of the derivation of the system (1-3) and corresponding boundary conditions see Kasahara and Puri (1981).

By the method of separation of the variables, the vector of 3D model variables $\left[u^{\prime}, v^{\prime}, h^{\prime}\right]^{\mathrm{T}}$ as functions of the longitude $(\lambda)$, latitude $(\varphi)$, vertical level $(\sigma)$ and time $t$ is represented as the product of $2 \mathrm{D}$ motions (their vector denoted $[u, v, h]$ and the vertical structure functions $G(\sigma)$ :

$\left[u^{\prime}, v^{\prime}, h^{\prime}\right]^{\mathrm{T}}(\lambda, \varphi, \sigma, t)=G(\sigma)[u, v, h]^{\mathrm{T}}(\lambda, \varphi, t)$.

The substitution of (5) into (1)-(3) leads to the vertical structure equation (VSE) and the horizontal structure equations (HSEs). The two systems of equations are connected by particular values of a separation parameter $D$ which is called the equivalent height. The vertical structure function $G(\sigma)$ is a solution of the VSE written in the dimensionless form as

$\frac{\mathrm{d}}{\mathrm{d} \sigma}\left(\frac{\sigma}{S} \frac{\mathrm{d} G(\sigma)}{\mathrm{d} \sigma}\right)+\frac{H_{*}}{D} G(\sigma)=0$,

where stability parameter $S(\sigma)=R \Gamma_{0} /\left(g H_{*}\right)$. We assume that $S>0$ for stable stratification. The parameter $H_{*}$ is a scaling constant with the dimension of height, $H_{*}=8 \mathrm{~km}$. The surface and top boundary conditions become 
$\frac{\mathrm{d} G(\sigma)}{\mathrm{d} \sigma}+\frac{\Gamma_{0}}{T_{0}} G(\sigma)=0$ at the surface, $\sigma=1$, and

$\sigma \frac{\mathrm{d} G(\sigma)}{\mathrm{d} \sigma}=0$ at some small value of $\sigma=\sigma_{T}$,

respectively. The top boundary condition applied at $\sigma_{T}$, as opposed to precisely zero, is a means of avoiding the issue of the VSE eigenvalue problem becoming singular. With boundary conditions (7-8), all eigenvalues i.e. equivalent height $D$ are positive, and the corresponding eigenfunctions are orthogonal in the sense that

$\int_{\sigma_{T}}^{1} G_{i}(\sigma) G_{j}(\sigma) \mathrm{d} \sigma=\delta_{i j}$

where $\delta_{i j}=1$ if $i=j$ and zero otherwise.

The HSEs are identical in form with the global shallow water equations having the water depth $D$ (Hough 1898; Kasahara 1976; Swarztrauber and Kasahara 1985). The horizontal motions $\left[u_{m}, v_{m}, h_{m}\right]^{\mathrm{T}}$ and time $t$ are made nondimensional by

$\tilde{u}_{m}=\frac{u_{m}}{\sqrt{g D_{m}}}, \quad \tilde{v}_{m}=\frac{v_{m}}{\sqrt{g D_{m}}}, \quad \tilde{h}_{m}=\frac{h_{m}}{D_{m}}, \quad \tilde{t}=2 \Omega t$.

As the non-dimensional HSEs are linear with respect to time, their solution $\left[\tilde{u}_{m}, \tilde{v}_{m}, \tilde{h}_{m}\right]^{\mathrm{T}}$ can be expressed in terms of harmonics in time as

$\mathbf{W}_{m}(\lambda, \varphi, \tilde{t})=\left[\tilde{u}_{m}, \tilde{v}_{m}, \tilde{h}_{m}\right]^{\mathrm{T}}=\mathbf{H}_{n}^{k}(\lambda, \varphi, m) e^{-i v_{n}^{k}(m) \tilde{t}}$,

where the subscript $m$ denotes the $m$-th vertical mode after the vertical projection followed by the normalization (10). The horizontal structure functions $\mathbf{H}_{n}^{k}(\lambda, \varphi, m)$, known as the Hough functions, depend on the zonal wavenumber $k$ and meridional index $n$ for every $m$ and the corresponding dimensionless frequency is denoted $v_{n}^{k}(m)$. The separation of the zonal and meridional dependencies and the periodic boundary conditions in the longitudinal direction lead to the following solution for $\mathbf{H}_{n}^{k}(m)$ for discrete values of $k$ in the form of the Hough harmonics:

$\mathbf{H}_{n}^{k}(\lambda, \varphi, m)=\boldsymbol{\Theta}_{n}^{k}(\varphi, m) e^{i k \lambda}$,

The meridional dependence is described by the Hough function vector $\boldsymbol{\Theta}$,

$\boldsymbol{\Theta}_{n}^{k}(\varphi, m)=\left[U_{n}^{k}(\varphi, m), i V_{n}^{k}(\varphi, m), Z_{n}^{k}(\varphi, m)\right]^{\mathrm{T}}$, with $U, V$ and $Z$ meridionally dependent profiles of the zonal velocity, meridional velocity and geopotential height, respectively, all characterized by the zonal wavenumber $k$ and meridional index $n$ for each $m$.

For every combination of the three indices $k, n$ and $m$, the dimensionless frequency $v_{n}^{k}(m)$ defines three eigensolutions of the horizontal oscillations. One of the solutions is a lowfrequency, westward-propagating Rossby wave, also denoted balanced mode. The other two solutions are high-frequency inertial gravity (IG) waves that propagate eastward or westward. For details of their derivation and the corresponding frequency relationships we refer the reader to Swarztrauber and Kasahara (1985) and Phillips (1990).

The derivation of solutions in the form (11) applies the following global inner product:

$\left\langle\mathbf{W}_{p}, \mathbf{W}_{r}\right\rangle=\frac{1}{2 \pi} \int_{0}^{2 \pi} \int_{-1}^{1}\left(\tilde{u}_{p}^{*} \tilde{u}_{r}+\tilde{v}_{p}^{*} \tilde{v}_{r}+\tilde{h}_{p}^{*} \tilde{h}_{r}\right) \mathrm{d} \mu \mathrm{d} \lambda$,

where $\mu=\sin (\varphi)$ and the asterisk (*) denotes the complex conjugate. Subscript $p$ refers to a particular mode corresponding to a zonal wavenumber $k_{p}$, a meridional index $n_{p}$ and a vertical mode index $m_{p}$, while subscript $r$ indicates another mode. The global orthogonality of the Hough functions for each $m$ can be written as

$\left\langle\mathbf{H}_{n}^{k}, \mathbf{H}_{n^{\prime}}^{k^{\prime}}\right\rangle=\frac{1}{2 \pi} \int_{0}^{2 \pi} \int_{-1}^{1}\left(\mathbf{H}_{n}^{k}\right)^{*} \cdot \mathbf{H}_{n^{\prime}}^{k^{\prime}} \mathrm{d} \mu \mathrm{d} \lambda=\delta_{n n^{\prime}} \delta_{k k^{\prime}}$.

Further details of the NMF derivation in the $\sigma$ coordinates are provided in Žagar et al. (2015) and references therein.

The NMF method has previously been applied in a number of studies to analyse atmospheric energetics (e.g. Tanaka 1985; Tanaka and Kung 1988; Tanaka and Kimura 1996; Terasaki and Tanaka 2007; Marques and Castanheira 2012), baroclinic instability (Kasahara and Tanaka 1989) and spatiotemporal variability (e.g. Tanaka and Kung 1989; Castanheira et al. 2002; Žagar et al. 2017; Blaauw and Žagar 2018) in addition to its extensive use for the initialization of NWP models (Machenhauer 1977; Daley 1991). In particular, Tanaka and Kung (1988) first considered stationary and transient component of the global total energy using NMFs. Castanheira et al. (2002) analyzed variability of the Northern Hemisphere winter circulation by combining the principal component analysis with time series of the Hough expansion coefficients of data on standard pressure levels. They demonstrated that selected normal modes data resemble well-known components of the Northern Hemisphere variability such as the observed winter North Atlantic Oscillation pattern. A more extensive set of their results was presented in Castanheira (2000).

All cited studies apply the NMF decomposition using the linearization around the state of rest. When the mean zonal flow is taken into account, the frequencies of wave solutions of the linearized global shallow water equations change and the frequency spectrum can become continuous, and solutions to such system barotropically unstable (Kasahara 1980). However, the structure of associated Hough functions does not change significantly in the case with the linearization around the non-zero mean zonal flow (see Corrigendum to Kasahara 
1980) implying that the NMFs for the reference state of rest can be reliably used for the spectral decomposition of the lowresolution data.

\subsection{Modal decomposition of discrete 3D data}

The NMF projection of global data available on $J$ terrain-following levels is performed using the MODES software (Žagar et al. 2015). The procedure consists of two steps. In the first step, the input data vector $\mathbf{X}\left(\lambda, \varphi, \sigma_{j}\right)$ at time step $t$ on $j$-th $\sigma$ level is decomposed using a series of $M$ orthogonal vertical structure functions $G_{m}(j)$ as

$\mathbf{X}\left(\lambda, \varphi, \sigma_{j}\right)=\sum_{m=1}^{M} G_{m}(j) \mathbf{S}_{m} \mathbf{X}_{m}(\lambda, \varphi)$,

where $\mathbf{S}_{m}$ is a $3 \times 3$ diagonal matrix with elements $\sqrt{g D_{m}}$, $\sqrt{g D_{m}}$, and $D_{m}$ for the purpose of normalization (10). The result of (15) is the vector $\mathbf{X}_{m}(\lambda, \varphi)=\left[\tilde{u}_{m}, \tilde{v}_{m}, \tilde{h}_{m}\right]^{\mathrm{T}}$ that describes non-dimensional oscillations of the horizontal wind and height fields in a shallow-water system with depth $D_{m}$. The vertical mode index $m$ varies from the external (barotropic) mode, $m=1$, to the total number of vertical modes $M$, with $M \leq J$.

In the second step, the dimensionless horizontal motions for each $m$ are represented by a series of the Hough harmonics $\mathbf{H}_{n}^{k}$ using the complex Hough expansion coefficients $\chi_{n}^{k}(m)$ as

$\mathbf{X}_{m}(\lambda, \varphi)=\sum_{n=1}^{R} \sum_{k=-K}^{K} \chi_{n}^{k}(m) \mathbf{H}_{n}^{k}(\lambda, \varphi ; m)$.

In (16), the parameter $K$ denotes the maximal number of zonal waves whereas the maximal number of meridional modes, denoted $R$, combines all of the Rossby modes, the eastward-propagating IG modes and the westward-propagating IG modes. The three indices $k, n$, and $m$ constitute the 3 -component modal index $v=(k, n, m)$ of the Hough expansion coefficients, $\chi_{v}$.

In practice, the 3D data expansion is performed by first computing $\mathbf{X}_{m}$ as

$\mathbf{X}_{m}(\lambda, \varphi)=\mathbf{S}_{m}^{-1} \sum_{j=1}^{J} G_{m}(j) \mathbf{X}\left(\lambda, \varphi, \sigma_{j}\right)$,

where we use (15) and the orthogonality (9) of discretized vertical structure functions. This is followed by the computation of the Hough expansion coefficients $\chi_{\nu}$ as

$\chi_{\nu}=\chi_{n}^{k}(m)=\frac{1}{2 \pi} \int_{0}^{2 \pi} \int_{-1}^{1}\left(\mathbf{H}_{n}^{k}\right)^{*} \cdot \mathbf{X}_{m} d \mu d \lambda$.
The zonal expansion is performed by using the fast Fourier transform while the Gaussian quadrature approximates the integration in the meridional direction. Numerical aspects of the horizontal expansion are described in Swarztrauber and Kasahara (1985). Equations (15) and (17) are the vertical transform pair whereas Eqs. (16) and (18) are the horizontal transform pair.

The result of the expansion (17-18) is the time series of the non-dimensional coefficients $\chi_{\nu}(t)$. The dimensional form of the derived statistical quantities is obtained by the multiplication implied by $\mathbf{S}_{m}$ for each $m$.

\subsection{Variability and bias indices}

With reanalyses and the climate model outputs available in terms of $k, n$ and $m$ indices, all moments can be evaluated in the space of normal modes for the Rossby and inertio-gravity waves. The evaluation of spatial variability in terms of energy spectra of kinetic energy and available potential energy is commonly applied to NWP models but also to reanalysis data (e.g. Žagar et al. 2017). Here we introduce definitions of temporal variability and bias using the same notation.

\subsubsection{Spatial variability}

The multiplication of the $\chi_{v}$ coefficients by $\left(g D_{m}\right)^{1 / 2}$ gives the total energy in mode $v=(k, n, m)$, denoted $I_{v}$ in units $\mathrm{m}^{2} / \mathrm{s}^{2}$ or $\mathrm{J} / \mathrm{kg}$ :

$I_{v}=I_{n}^{k}(m)=\frac{1}{2} g D_{m} \chi_{n}^{k}(m)\left[\chi_{n}^{k}(m)\right]^{*}$.

Energy for the $m$ th vertical mode is obtained by summing over all horizontal modes as

$I_{m}=\frac{1}{2} g D_{m} \sum_{n=1}^{R} \sum_{k=-K}^{K} \chi_{n}^{k}(m)\left[\chi_{n}^{k}(m)\right]^{*}$

which is equivalent to a sum of the specific kinetic and available potential energy, $K_{m}+P_{m}$ (Žagar et al. 2015):

$K_{m}+P_{m}=\frac{1}{2}\left(u_{m}^{2}+v_{m}^{2}\right)+\frac{1}{2} \frac{g}{D_{m}} h_{m}^{2}$.

Most of time, we are interested in the meridionally and vertically integrated energy which is computed as

$I_{k}=\frac{1}{2} \sum_{m=1}^{M} g D_{m} \sum_{n=1}^{R} \chi_{n}^{k}(m)\left[\chi_{n}^{k}(m)\right]^{*}$.

The spectrum $I_{k}$ at a single time instant describes how energy is distributed as a function of the zonal wavenumber. Long-term average of energy is $\overline{I_{k}}=1 / N \sum_{t=1}^{N} I_{k}(t)$, with the length of the time series denoted $N$. The average 
energy spectra for the model simulation and reanalysis data are denoted $\overline{I_{k}^{c}}$ and $\overline{I_{k}^{a}}$, respectively. Notice that $\bar{I}_{k}$ is different from the energy spectra of the mean circulation obtained by computing the energy spectrum from the average Hough expansion coefficients, $\overline{\chi_{v}}$,

$\overline{\chi_{\nu}}=\frac{1}{N} \sum_{t=1}^{N} \chi_{\nu}(t)$.

In fact, it is easy to show that for each $v$ the difference between the energy of the time mean component $\overline{\chi_{v}}$, $I\left(\overline{\chi_{v}}\right)=1 / 2 g D_{m} \overline{\chi_{v}}\left[\overline{\chi_{v}}\right]^{*}$, and the time mean energy $\overline{I_{k}}$ corresponds to the transient circulation energy in mode $v, T_{v}$

$T_{\nu}=\frac{1}{N} \sum_{t=1}^{N} \frac{1}{2} g D_{m}\left(\chi_{\nu}(t)-\overline{\chi_{\nu}}\right)\left(\chi_{\nu}(t)-\overline{\chi_{\nu}}\right)^{*}$,

i.e. $\overline{I_{v}}=I\left(\overline{\chi_{v}}\right)+T_{v}$.

\subsubsection{Spectrum of bias}

For a single mode $v$, we denote the complex expansion coefficients for the climate model and verifying analysis $\chi_{v}^{c}$ and $\chi_{v}^{u}$, respectively. Their time-averaged difference is computed as

$\overline{\Delta \chi_{\nu}}=\frac{1}{N} \sum_{t=1}^{N}\left[\chi_{\nu}^{c}(t)-\chi_{\nu}^{a}(t)\right]=\overline{\chi_{\nu}^{c}}-\overline{\chi_{\nu}^{a}}$.

If differences between the model and analysis were random with zero mean, their time average should be close to zero. A systematic difference, however, is referred to as a bias. Equation (25) thus formally defines model bias in every zonal wavenumber, meridional and vertical mode. For any $v$ or a range of indices, $\overline{\Delta \chi_{\nu}}$ can be filtered from modal space back to physical space by applying (15-16). As the NMF decomposition is complete, filtering of all modes should ideally correspond to the averaged difference in physical space for the wind components, geopotential and surface pressure fields. In the following, we prove validity of this statement.

By multiplying the amplitudes of bias coefficients $\overline{\Delta \chi_{\nu}}$ by a factor $g D_{m}$ we obtain the spectrum of bias variance. In order to see this, we define specific modal bias variance $B_{v}$ for a single mode $v$ as

$B_{v}=g D_{m} \overline{\Delta \chi_{v}}\left[\overline{\Delta \chi_{\nu}}\right]^{*}$.

The variance of bias at the point $\left(\lambda_{i}, \varphi_{j}, m\right)$ is defined as

$Q\left(\lambda_{i}, \varphi_{j}, m\right)={\overline{\Delta u_{m}}}^{2}+{\overline{\Delta v_{m}}}^{2}+\frac{g}{D_{m}}{\overline{\Delta h_{m}}}^{2}$,

with bias for individual variables $u_{m}, v_{m}$ and $h_{m}$ at point $\left(\lambda_{i}, \varphi_{j}, m\right)$ defined as (for the zonal wind)
$\overline{\Delta u_{m}}=\frac{1}{N} \sum_{t=1}^{N}\left[u_{m}^{c}(t)-u_{m}^{a}(t)\right]=\overline{u_{m}^{c}}-\overline{u_{m}^{a}}$,

and similarly for $v_{m}$ and $h_{m}$.

We can show that the summation of Eq. (26) over all $(k, n)$ is equivalent to the horizontal integral of the squares of the biases in wind and geopotential height fields after the vertical projection, as defined by Eq. (27), that is calculated as a double summation using the Gaussian weights $w\left(\lambda_{i}, \varphi_{j}\right)$ :

$\sum_{k} \sum_{n} B_{n}^{k}(m) \approx \sum_{i} \sum_{j} w\left(\lambda_{i}, \varphi_{j}\right) Q\left(\lambda_{i}, \varphi_{j}, m\right)$.

To show (29), we use (16) and (17) to write the horizontal expansion for a difference between the model and reanalysis after the vertical projection, $\Delta \mathbf{X}_{m}=\mathbf{X}_{m}^{c}-\mathbf{X}_{m}^{a}$, in terms of $\Delta \chi_{n}^{k}(m)=\Delta \chi_{v}=\chi_{v}^{c}-\chi_{\nu}^{a}$, and average it over time series of $\mathrm{N}$ differences to obtain

$\overline{\Delta \mathbf{X}_{m}}=\sum_{n=1}^{R} \sum_{k=-K}^{K} \overline{\Delta \chi_{n}^{k}(m)} \mathbf{H}_{n}^{k}$

Then we take the inner product (13) of expression (30) with itself and multiply from the left by $g D_{m}$ and apply (10) to obtain

$$
\begin{aligned}
g D_{m} & \left\langle\overline{\Delta \mathbf{X}_{m}}, \overline{\Delta \mathbf{X}_{m}}\right\rangle \\
& =\frac{1}{2 \pi} \int_{0}^{2 \pi} \int_{-1}^{1}\left({\overline{\Delta u_{m}}}^{2}+{\overline{\Delta v_{m}}}^{2}+\frac{g}{D_{m}}{\overline{\Delta h_{m}}}^{2}\right) d \mu d \lambda .
\end{aligned}
$$

Applying the orthogonality of the Hough harmonics (14) the right-hand side of (31) becomes

$\sum_{n=1}^{R} \sum_{k=-K}^{K} g D_{m} \overline{\Delta \chi_{v}}\left[\overline{\Delta \chi_{\nu}}\right]^{*}$

the horizontally integrated specific modal bias variance (26), i.e. the left-hand side of (29). Using the Gaussian weights to calculate the right-hand side of (29), we obtain the righthand side of Eq. (27).

\subsubsection{Temporal variability}

Variability is the square root of variance which for a single mode $v$ is denoted $V_{v}=V_{n}^{k}(m)$. Unbiased variance is computed for the GCM and reanalysis data as

$V_{v}=\frac{1}{N-1} \sum_{t=1}^{N} g D_{m}\left(\chi_{\nu}(t)-\overline{\chi_{\nu}}\right)\left(\chi_{\nu}(t)-\overline{\chi_{\nu}}\right)^{*}$.

Unit of $V_{v}$ is $\mathrm{m}^{2} / \mathrm{s}^{2}$. Notice that the difference to Eq. (24) is the factor $1 / 2$ and the normalization by $(N-1)$ instead of $N$; 
in other words, if we use the biased variance, the following relation applies:

$\overline{I_{v}}-I\left(\overline{\chi_{v}}\right)=\frac{1}{2} V_{v}$.

Using Eq. (34) to evaluate $\overline{I_{v}^{c}}-\overline{I_{v}^{a}}$ and expressing $I\left(\overline{\chi_{v}^{c}}\right)$ as $I\left(\overline{\chi_{v}^{c}}\right)=I\left(\overline{\Delta \chi_{\nu}}+\overline{\chi_{v}^{a}}\right)$, we arrive at the following expression coupling the bias with variance and energy distribution in the model and verifying analyses:

$\overline{I_{v}^{c}}-\overline{I_{v}^{a}}=\frac{1}{2}\left[V_{v}^{c}-V_{v}^{a}\right]+\frac{1}{2} B_{v}+P\left(\overline{\Delta \chi_{v}}, \overline{\chi_{v}^{a}}\right)$,

with the covariance term $P\left(\overline{\Delta \chi_{\nu}}, \overline{\chi_{\nu}^{a}}\right)$ defined as

$$
\begin{aligned}
P\left(\overline{\Delta \chi_{v}}, \overline{\chi_{v}^{a}}\right) & =\frac{1}{2} g D_{m}\left(\overline{\Delta \chi_{v}}\left[\overline{\chi_{v}^{a}}\right]^{*}+\left[\overline{\Delta \chi_{v}}\left[\overline{\chi_{v}^{a}}\right]^{*}\right]^{*}\right) \\
& =g D_{m}\left(\left[\overline{\Delta \chi_{\nu}}\right]_{r}\left[\overline{\chi_{v}^{a}}\right]_{r}+\left[\overline{\Delta \chi_{\nu}}\right]_{i}\left[\overline{\chi_{v}^{a}}\right]_{i}\right),
\end{aligned}
$$

where the subscripts $r$ and $i$ denote the real and imaginary parts, respectively. The term $P\left(\overline{\Delta \chi_{v}}, \overline{\chi_{v}^{a}}\right)$ describes the covariance between the mean state of the verifying reanalysis and bias in the same mode. Using (34) for the model and reanalysis, Eq. (35) can be rewritten as

$I^{c}\left(\overline{\chi_{v}}\right)-I^{a}\left(\overline{\chi_{v}}\right)=\frac{1}{2} B_{v}+P\left(\overline{\Delta \chi_{v}}, \overline{\chi_{v}^{a}}\right)$.

Equation (35) states that deficiency in simulated mean energy in mode $v$ can be expressed as a sum of three terms: deficiency in simulated variance, the bias variance and the covariance between the mean state of verifying reanalysis and the bias in the same mode $v$. Equation (37) states that the misrepresentation of the climatological spatial variance (energy of the mean state) in the model with respect to the reanalysis is described by the 2-term bias expression that accounts for differences between the model and reanalysis in the variance and in the mean total energy.

Globally integrated squared variability defined by Eq. (33) is equivalent to the integral in physical space (after the vertical projection) of the variance $S_{m}$ which at the point $\left(\lambda_{i}, \varphi_{j}, m\right)$ is defined as

$S_{m}\left(\lambda_{i}, \varphi_{j}\right)=\operatorname{Var}\left(u_{m}\right)+\operatorname{Var}\left(v_{m}\right)+\frac{g}{D_{m}} \operatorname{Var}\left(h_{m}\right)$.

The three elements of the summation (38), $\operatorname{Var}\left(u_{m}\right)$, $\operatorname{Var}\left(v_{m}\right)$ and $\operatorname{Var}\left(h_{m}\right)$, denote variances of the wind components and modified geopotential height after the vertical projection; for example, interannual variance in zonal wind, $\operatorname{Var}\left(u_{m}\right)$, at the location $\left(\lambda_{i}, \varphi_{j}, m\right)$ is given by $\operatorname{Var}\left(u_{m}\right)=1 /(N-1) \sum_{t=1}^{N}\left(u_{m}(t)-\bar{u}_{m}\right)^{2}$, where $\bar{u}_{m}$ is the mean zonal wind at the location. The equivalence between $\sum_{k} \sum_{n} \sum_{m} V_{n}^{k}(m) \quad$ and $\quad \sum_{i} \sum_{j} w\left(\lambda_{i}, \varphi_{j}\right) \sum_{m} S_{m}\left(\lambda_{i}, \varphi_{j}\right)$.

can be easily shown following the proof of the equivalence between the modal and physical space bias, with the time averaging of differences replaced by the time averaging of squared departures from the time mean values.

\section{Simulations and reanalysis data}

\subsection{GCM simulations}

Numerical simulations are performed by SPEEDY model which is described in Molteni (2003) and Kucharski et al. (2006). It is a hydrostatic spectral GCM, built on the GFDL dynamical core (Held and Suarez 1994), with vorticity and divergence as the main prognostic variables, together with temperature, specific humidity and surface pressure. Convection, condensation, clouds, radiation, momentum and energy surface fluxes, and vertical diffusion are assessed by simplified parametrization schemes described in Molteni (2003).

We used SPEEDY version 41, with 8 vertical sigma levels and spectral truncation T30, which approximately corresponds to the resolution of $3.75^{\circ} \times 3.75^{\circ}$. Vertical levels in SPEEDY are at $\sigma=0.025,0.095,0.2,0.34,0.51,0.685$, 0.835 , and 0.95 (denoted L8). The horizontal hyperdiffusion on $\sigma$ surfaces is applied to vorticity, divergence, temperature and specific humidity at all wavenumbers in the form of the fourth-power Laplacian including a corrective term for temperature and specific humidity which simulates diffusion on pressure to avoid a spurious diffusion over topography. There is an additional linear damping at the model top levels applied to the zonal wind and a vertical diffusion applied to temperature (Kucharski, pers. comm.).

The prescribed monthly SST from reanalysis is assumed valid on 15th day of the month and daily SST value is linearly interpolated in between. Alternatively, the SST can be computed using a slab ocean thermodynamic mixed-layer model with a time-constant depth varying as the third power of cosine from $40 \mathrm{~m}$ in the tropics to $60 \mathrm{~m}$ in the extratropics. Due to the formulation of the model, the application of the slab ocean still requires the user to provide prescribed monthly mean SSTs and climatological net heat fluxes into the ocean. Computations of daily corrections to SST simulate a mixed layer of constant depth and heat capacity, taking into account the deviations of net heat fluxes into the ocean from the climatological ones (Herceg-Bulić and Kucharski 2012; Sun et al. 2017). The detailed SPEEDY version 41 documentation can be found at the website http://users.ictp. it/ kucharsk/speedy-net.html. 
SPEEDY performance has been evaluated in studies of teleconnections and ENSO (e.g. Polo et al. 2014; Sun et al. 2017), ocean-atmosphere interactions (e.g. Herceg-Bulić and Kucharski 2013), monsoon variability and precipitation (Feudale and Kucharski 2013) and extreme climate events (Scaife et al. 2009).

Two long model simulations were carried out. The first simulation was forced with monthly fields of SST from ERA-20C from January 1949 to December 2010. The second simulation used SPEEDY coupled to the slab ocean model (S1O) as described above. The two T30L8 datasets are denoted SPEEDY and SPEEDY-S1O, respectively. Both experiments started from atmosphere at rest on 1 January 1949 and cover the period until 31 December 2010. The outputs are saved as monthly mean values for all prognostic parameters and monthly accumulation for precipitation. The first 10 years are not used so that presented results are based on the 50-year period between January 1961 and December 2010.

\subsection{ERA-20C reanalysis data and comparison with SPEEDY}

The ERA-20C reanalysis dataset is described in Poli et al. (2016). The assimilated observations are surface pressure and surface winds over oceans, whereas the sea-surface temperature and sea-ice cover are from HadISST2 (Rayner et al. 2006). The ERA-20C analysis is forced by historical record of changes in climate forcing factors: greenhouse gases, volcanic aerosols and solar variations.

Monthly mean fields of ERA-20C over the period 1961-2010 were prepared at T30 horizontal resolution and 91 model levels from the ECMWF data archive. The transformed geopotential height defined in Sect. 2 is computed on model levels. Then the wind and geopotential height fields are vertically interpolated to the eight sigma levels used in SPEEDY.

The precipitation climatology in ERA-20C can be compared with the two simulations in Fig. 1. Overall the precipitation pattern in SPEEDY is simulated reasonably well in comparison with ERA-20C given that the average precipitation is a target of the model tuning when forced by the observed SST (Kucharski, pers. comm.). However, the precipitation amount and the regional and seasonal details are less satisfactory. Precipitation maxima in the tropics are overestimated, especially in the coupled-model run over the ITCZ in both Pacific and Atlantic. SPEEDY-SIO underestimates precipitation in the western south Pacific convergence zone and in the southern hemisphere (SH) subtropics. For the sake of model bias discussion later on, we mention also interannual variability. In ERA-20C, interannual variability of precipitation is maximal in the western and central Pacific in relation to ENSO. Variability simulated by SPEEDY in the same region is somewhat overestimated whereas SPEEDY-S1O does not contain the observed variability maximum due to missing ENSO variability (not shown).

The time-averaged, zonally-averaged tropospheric zonal winds in four seasons are shown in Fig. 2 for SPEEDY and ERA-20C along with their time averaged difference (bias). Averaged winds simulated by SPEEDY agree with the reanalysis reasonably well up to $200 \mathrm{hPa}$. The model has too strong subtropical jets above $400 \mathrm{hPa}$ as well as too strong tropical upper troposphere easterlies compared to ERA-20C. Stratospheric wind maxima, seen in ERA-20C, are not simulated by the model due to a poorly resolved stratosphere and damping.

These deficiencies become more pronounced in SPEEDY-SIO (not shown). The comparison of interannual variability reveals that the model on average significantly underestimates wind variability present in ERA-20C data. An exception is the SH midlatitude upper-troposphere zonal wind variability which is overestimated as noted also in the previous studies (Molteni 2003). This might be associated with a greater variability in the surface-pressure field over Antarctica in SPEEDY than in ERA-20C (not shown); surface pressure is a part of the total variability in modal space through the modified geopotential height. We also note that the model lacks the zonal wind variability in the tropical stratosphere, which is the maximal variability (a) ERA-20C

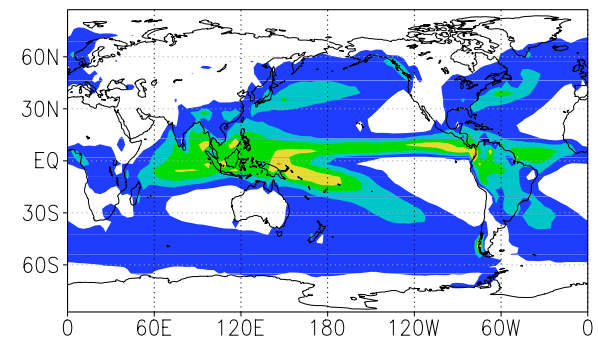

(b) SPEEDY

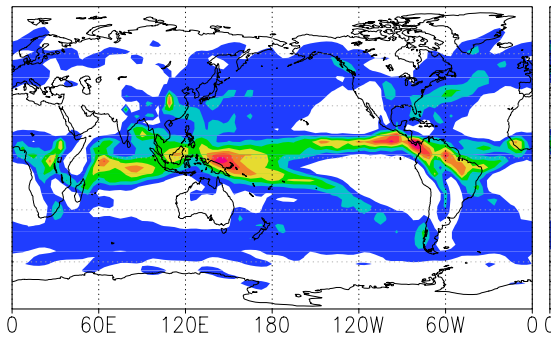

(c) SPEEDY-SIO

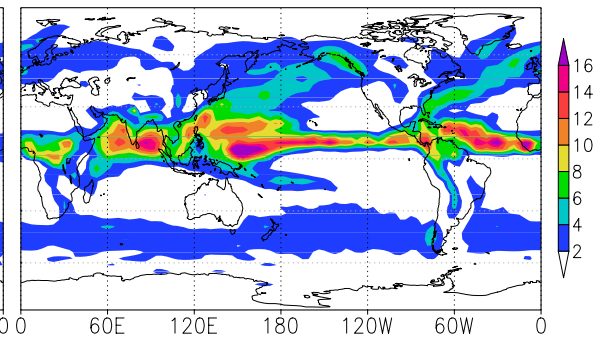

Fig. 1 Precipitation climatology based on 1961-2010 period for a ERA-20C, b SPEEDY and c SPEEDY-SlO in mm/day 
(a) ERA-20C mean, MAM

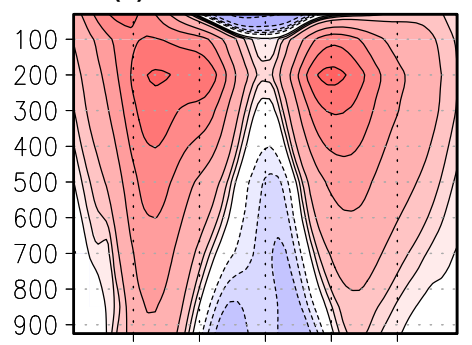

(e) SPEEDY mean, MAM (b) ERA-20C mean, JJA

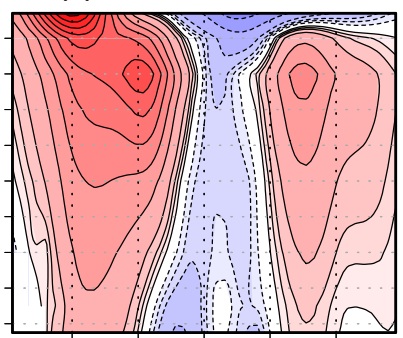

(f) SPEEDY mean, JJA
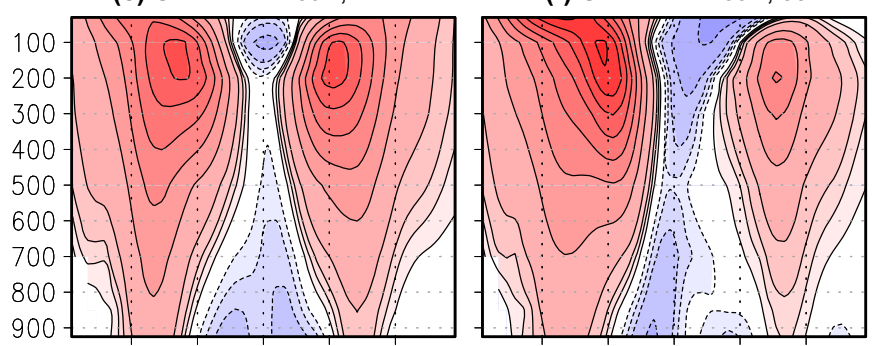

(c) ERA-20C mean, SON

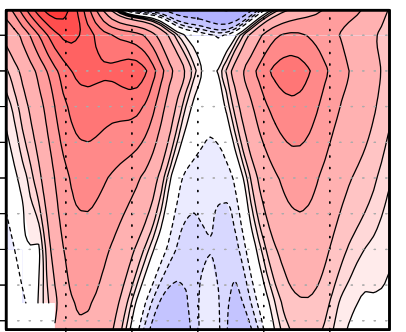

(g) SPEEDY mean, SON
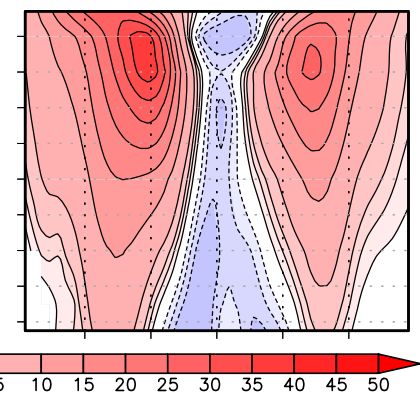

(i) SPEEDY bias, MAM

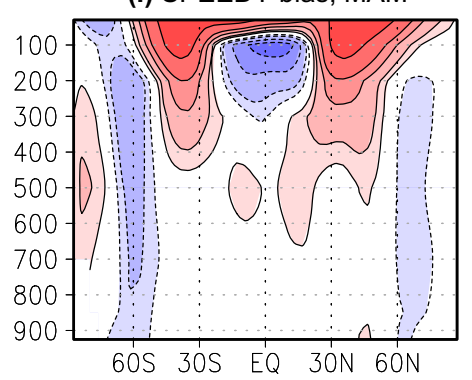

(j) SPEEDY bias, JJA

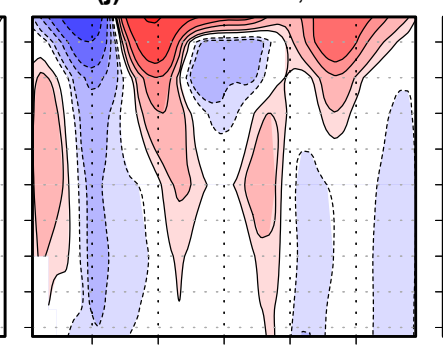

6OS 30S EQ 30N 60N

(k) SPEEDY bias, SON

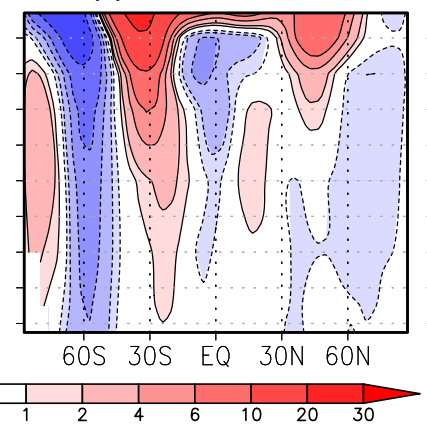

(d) ERA-20C mean, DJF

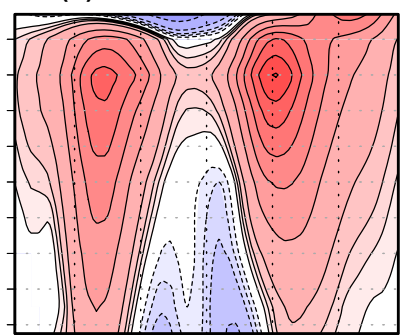

(h) SPEEDY mean, DJF

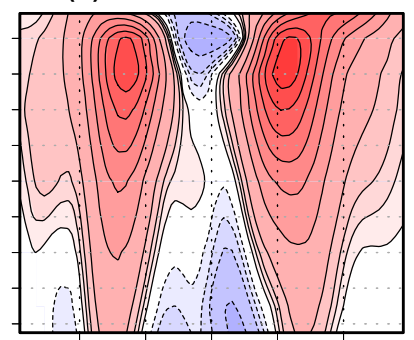

(I) SPEEDY bias, DJF

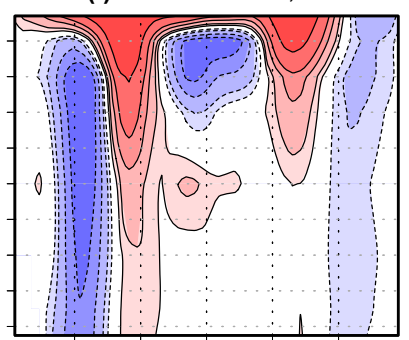

6OS 30S EQ 30N 60N

Fig. 2 Zonal mean zonal winds in a-d ERA-20C reanalysis data and $\mathbf{e}-\mathbf{h}$ SPEEDY, and $\mathbf{i}-\mathbf{l}$ SPEEDY biases against ERA-20C in the four seasons, all in $\mathrm{m} / \mathrm{s}$. From left to right: MAM, JJA, SON and DJF

in the ERA-20C dataset. This contributes a large part of the bias in the zonal mean state above $300 \mathrm{hPa}$ (Fig. 2i-1).

\subsection{Setup of the modal decomposition}

The expansion (15) is applied to SPEEDY and ERA-20C monthly outputs from January 1961 to December 2010, a total of 600 samples. The computation of vertical structure functions necessitates global temperature and stability vertical profiles which are calculated from ERA-20C. The difference between the globally averaged profiles for ERA-20C and SPEEDY is sufficiently small to produce no significant difference in the results of the vertical structure equation. The Hough functions are computed for every vertical mode, from $m=1$ to $M=8$. The applied horizontal truncations are $K=30$ and $R=90$ for the number of zonal wavenumbers and meridional modes, respectively. Ninety meridional modes include equal number (30) of the Rossby, eastward and westward inertio-gravity modes. For example, the structures of four horizontal modes for the barotropic vertical mode $m=1$ with the equivalent depth close to 10 $\mathrm{km}$, and zonal wavenumber $k=1$ are shown in Fig. 3. They include two frequently analyzed waves of the global atmosphere, the Kelvin wave ( $n=0$ eastward-propagating inertiogravity mode) and the $n=1$ Rossby wave.

The input data to the NMF projection are on regular Gaussian grid N24 which corresponds to $96 \times 48$ points in physical space. The hydrostatic geopotential height on model levels is computed from temperature data starting from the orography as a lower boundary and the background value obtained using the globally constant temperature at every level is subtracted. Similarly the pressure term in the computation of the modified geopotential height variable is reduced by the factor computed using the globally constant surface pressure value $1000 \mathrm{hPa}$ (Staniforth et al. 1985). Both ERA-20C and SPEEDY datasets were subjected to the same spectral filtering following Navarra et al. (1994) to 
(a) Rossby $\mathrm{n}=1$

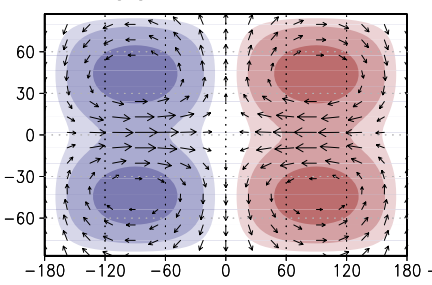

(b) Rossby $\mathrm{n}=2$

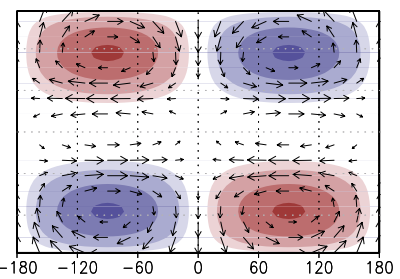

(c) Rossby $n=3$

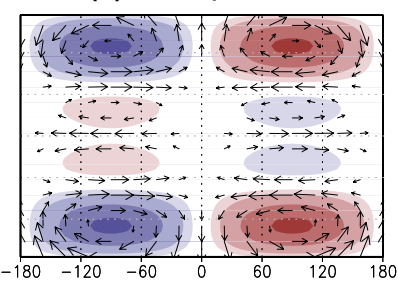

(d) Kelvin wave

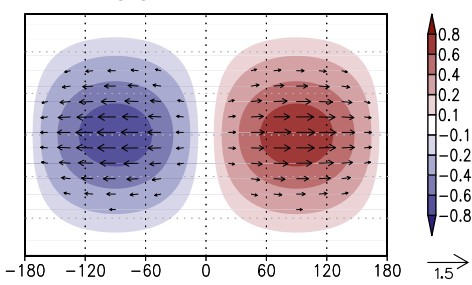

Fig. 3 Horizontal structure functions for the zonal wavenumber $k=1$ and vertical mode $m=1$. Shown are Rossby waves with meridional modes $\mathbf{a} n=1$, b $n=2$, $\mathbf{c} n=3$ and $\mathbf{d}$ the eastward-propagating inertio-gravity mode $n=0$ (Kelvin wave). Red and blue shades correspond to positive and negative values of the geopotential height in meters. Winds are in $\mathrm{m} / \mathrm{s}$ reduce Gibbs oscillations. The same filtering was applied to their orography fields before computing geopotential.

Seasonal means for each coefficient mode $v$ are obtained from (23) as averages over three monthly values for MAM, JJA, SON and DJF, and annual means as averages over the 12 calendar months. Seasonal and annual climatologies are defined by the fifty-year (number of samples is $N=50$ ) averages of seasonal and annual means, respectively, except for the northern hemisphere $(\mathrm{NH})$ winter (DJF) climatology which is computed from 49 years, $N=49$.

\section{Scale-dependent model validation}

First we present simulated energy spectra and variability in comparison to ERA-20C, then we evaluate biases in relation to variability and finally, we present some properties of the annual energy cycle in the model. It is worth mentioning that the same analysis was performed using the ERA-Interim data for period 1981-2010 and the results were very similar.

\subsection{Energy spectra}

For every monthly data, we evaluate Eq. (22) for the zonal distribution of total kinetic and available potential energy as

$E_{k}=\left(2-\delta_{k 0}\right) I_{k}=\left(2-\delta_{k 0}\right) \sum_{m=1}^{M} \frac{1}{2} g D_{m} \sum_{n=1}^{R}\left|\chi_{n}^{k}(m)\right|^{2}$,

where $\delta_{k 0}=1$ for $k=0$ and 0 otherwise, since zonal wavenumbers $k$ are stored for $0 \leq k \leq N_{k}$.

Figure 4 shows the average energy distribution in ERA$20 \mathrm{C}$ and in the two SPEEDY simulations in the annual means and in DJF and JJA seasons. Each panel includes information on the percentage of energy in waves with respect to the total energy, i.e. $100 \% \sum_{k \neq 0} I_{k} / \sum_{k} I_{k}$. It shows that there is about (a) Annual

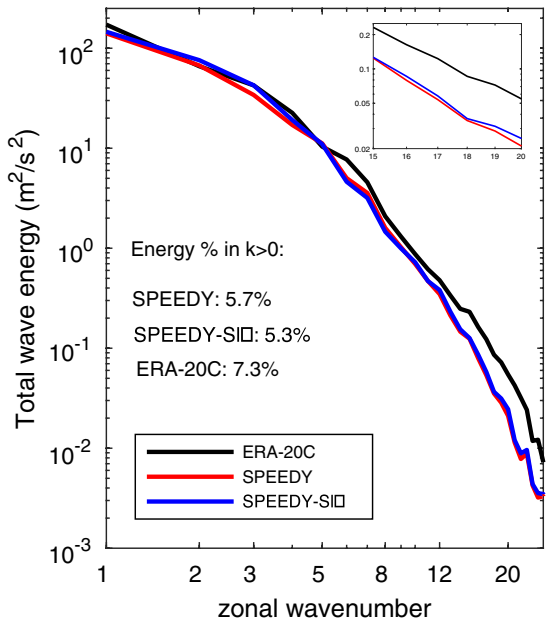

(b) JJA

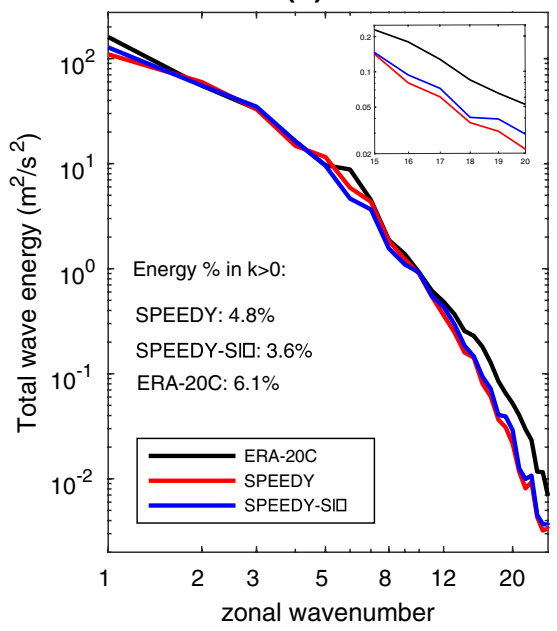

(c) DJF

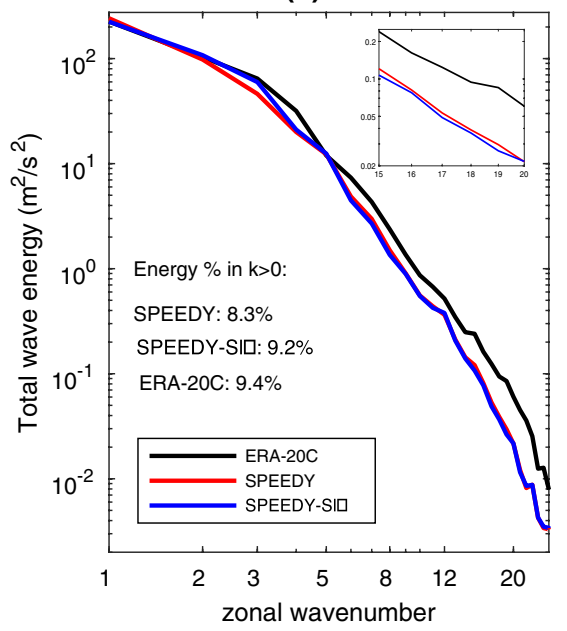

Fig. 4 Average energy distribution as a function of the zonal wavenumber in ERA-20C, SPEEDY and SPEEDY-SIO datasets. a Annual climatological spectra, b JJA and c DJF seasonal climatology energy spectra as defined by Eq. (10). Numbers in each panel show the percentage of energy in waves for each dataset. Small interior figures are zooms for $k=15-20$ 
$50 \%$ more energy in waves in the $\mathrm{NH}$ winter than in summer in ERA-20C, and that differences between the seasons are largest at large scales. On average, our low-resolution model lacks wave motions and the gap between the reanalysis and model is smaller in DJF than in JJA, probably in relation to stronger wave dynamics in NH. As the scale reduces, the relative difference between SPEEDY and ERA-20C increases. In the wavenumbers range $k=15-20$, the model has half or less energy than ERA-20C (zoom in each panel Fig. 4); for example, in DJF, SPEEDY has $50 \%$ of ERA-20C energy in $k=15,42 \%$ in $k=17$ and only $36 \%$ in $k=20$. Differences between SPEEDY and SPEEDY-SIO are largest at planetary scales, especially in DJF in $k=3$ (about $30 \%$ ).

So far climate models have not been systematically validated with reanalyses using energy spectra. In order to relate presented differences in energy spectra with the model shortcomings, it is useful to discuss them in relation to other properties of the model i.e. temporal variability.

\subsection{Interannual variability}

The zonal spectrum of variability is computed as the square root of the zonal variance spectrum after the vertical and meridional integration of variance computed as deviations of the seasonal and annual means from their respective climatologies using Eq. (33). The resulting variability spectrum is

$\mathcal{V}_{k}=\sqrt{V_{k}}=\sqrt{\left(2-\delta_{k 0}\right) \sum_{n=1}^{R} \sum_{m=1}^{M}\left[V_{n}^{k}(m)\right]^{2}}$,

where $\delta_{k 0}=1$ for $k=0$ and 0 otherwise $\left(\mathcal{V}_{k}\right.$ is presented only for positive $k$ ). Note here that variability values apply per zonal grid point as the applied forward Fourier transformation pair in Eq. (16) includes normalization by the number of grid points along the latitude circle.
Interannual variability obtained by Eq. (41) is shown in Fig. 5 for the zonal wavenumber $k=0-10$ as the total variability and variability divided in the symmetric and asymmetric components with respect to the equator. The two components are the square roots of the symmetric and asymmetric variance which are defined by the value of the meridional mode $n$. The symmetric Rossby and inertiogravity modes have odd and even, respectively, values of $n$ and their geopotential height and zonal winds are symmetric with respect to the equator, as illustrated in Fig. 3. Opposite applies to the asymmetric modes.

Figure 5 shows a strong dependence of the interannual variability on the zonal scale in the model and reanalysis; that is, the larger the scale, the greater the variability. However, the model significantly underestimates variability presented in ERA-20C; the underestimation in $k=0$ is about one-third but it decreases as $k$ increases. The underestimation of large-scale variability is predominantly in the symmetric component, especially in $k=0$ where SPEEDY accounts for only about $64 \%$ of the symmetric variability in ERA-20C. For each $k>0$, SPEEDY-SIO contains less variability than the SST-forced run (except in the asymmetric part of $k=0$ ) although the difference becomes small for $k>10$. The difference between the two simulations at large scales is smaller for the asymmetrical than for symmetric component. In ERA-20C, the symmetric component of the zonal mean circulation is the largest contributor to the global variance (about $37 \%$ for our T30L8 dataset). The contributions of the symmetric and asymmetric variance become nearly equal at wavenumber $k=4$.

Figure 6 shows integrated interannual variance and its normalized spectra. The amplitude of integrated variance of the 8-level ERA-20C dataset on T30 resolution is around $53 \mathrm{~m}^{2} / \mathrm{s}^{2}$ (Fig. 6a), i.e. about $7.3 \mathrm{~m} / \mathrm{s}$. Quantitatively SPEEDY produces about $60 \%$ and SPEEDY-SIO around 50\% variability in ERA-20C (Fig. 6c). The zonal (a) Interannual variability

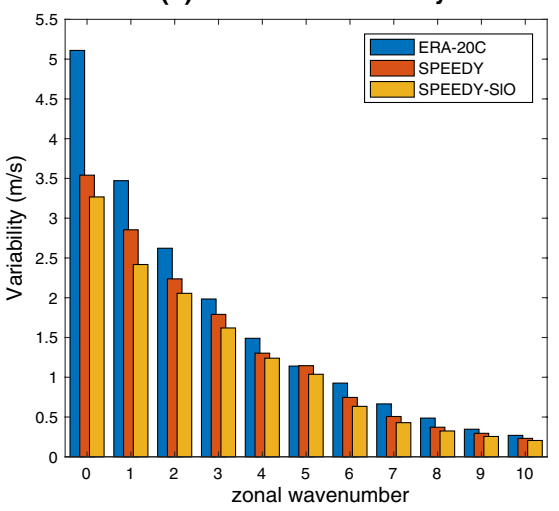

(b) Symmetric interannual variability

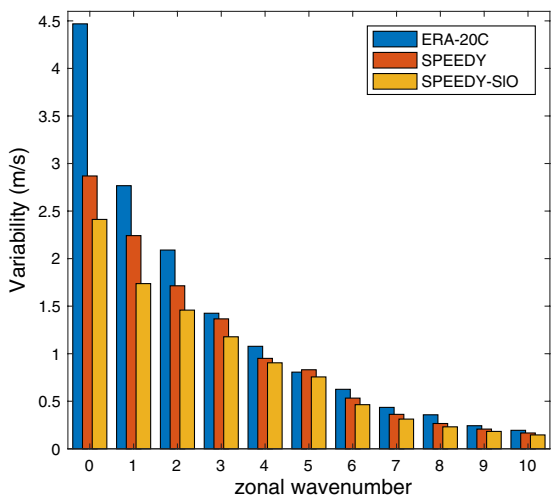

(c) Asymmetric interannual variability

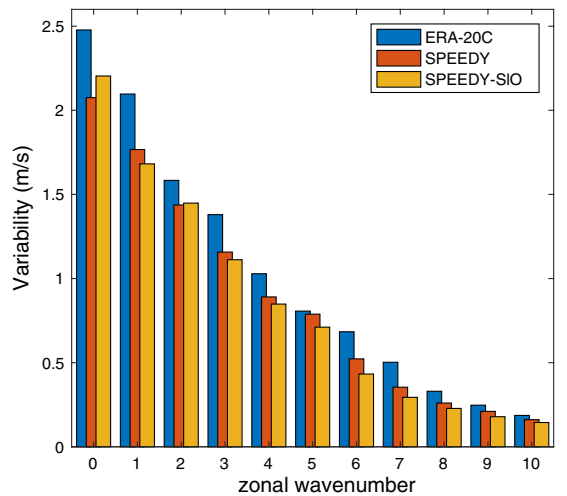

Fig. 5 Interannual variability of annual means as a function of the zonal wavenumber. a Variability $\mathcal{V}_{k}$ computed by Eq. (41), and its $\mathbf{b}$ symmetric and $\mathbf{c}$ asymmetric components. Units are $\mathrm{m} / \mathrm{s}$ 
(a) Cumulative variance

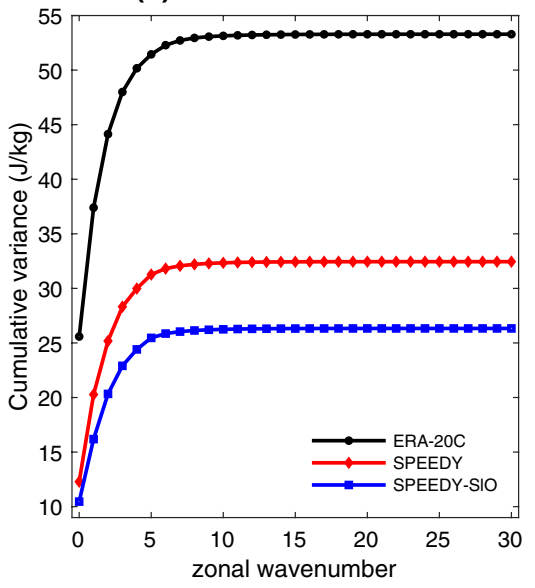

(b) Cumulative relative variance

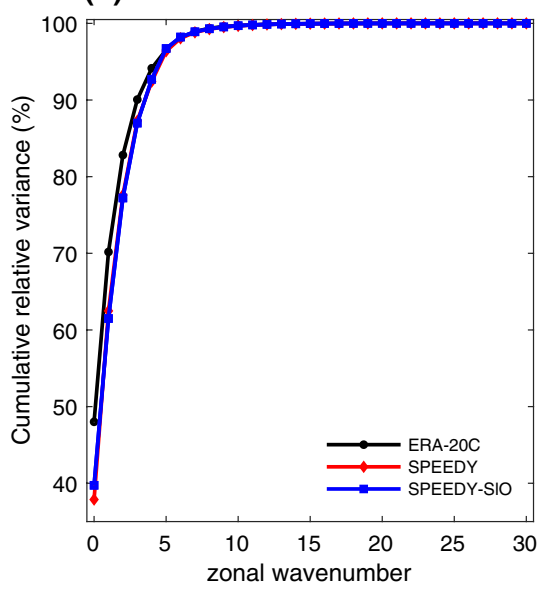

(c) Cumulative wrt ERA-20C

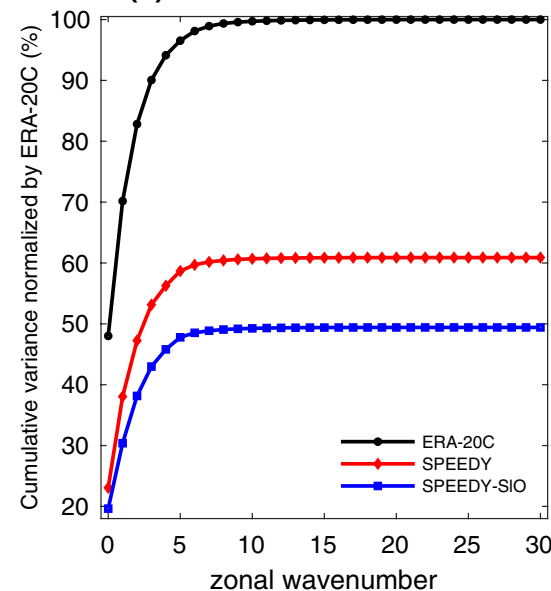

Fig. 6 Cumulative interannual variance in three datasets: ERA-20C reanalysis (black curve), SPEEDY forced by SST from ERA-20C (red curve) and SPEEDY-SIO which is using a slab-ocean model (blue curve). a Global cumulative variance. For each zonal wavenumber $k$,
$V_{k}$ is summed up for all smaller $k$. $\mathbf{b}$ As in $\mathbf{a}$, but variance in each $k$ is scaled by the total variance in the same dataset. $\mathbf{c}$ As in $\mathbf{b}$, but each dataset is scaled by the total variance in ERA-20C

total variance amplitude makes $60 \%$ of that in ERA-20C (Fig. 7d). The total simulated variance by SPEEDY-SIO varies a lot among the seasons with respect to ERA-20C, from $\sim 47 \%$ in SON to about $\sim 90 \%$ in MAM.

Similar to Fig. 5, we can discuss interannual seasonal variance in symmetric and asymmetric components. For $k>4$, the march of seasons is seen in greater asymmetric than symmetric variance in ERA-20C in wavenumbers $k=$ 5-7, 5-6, 7 and 6-7 in MAM, JJA, SON and DJF seasons, respectively (figures not shown). This is likely a result of a stronger interannual variance in the baroclinic activity of the midlatitude storm tracks in various seasons. There is a lack of symmetric variance at large scales in the model that is especially strong at planetary scales in SPEEDY-SIO.

The missing variance appears to be in odd Rossby meridional modes, particularly in $n=3$ Rossby mode (shown in Fig. 3c). Instead, the model has largest variance in the asymmetric Hough mode $n=2$, especially large in the coupled run (not shown). Variability in small zonal and meridional scales is poorly represented in the model; the discussed lack the smallest variance is also in JJA when SPEEDY and

Table 1 Global interannual variance integrated vertically over 8 terrain-following levels $(\sigma=0.025,0.095,0.2,0.34,0.51,0.685,0.835,0.95)$, meridionally and for the selected zonal wavenumbers on T30 horizontal grid

\begin{tabular}{lllllllllll}
\hline$k$ & Ann E & Ann S & MAM E & MAM S & JJA E & JJA S & SON E & SON S & DJF E & DJF S \\
\hline 0 & 48 & 38 & 40 & 36 & 40 & 28 & 45 & 27 & 39 & 33 \\
$0-1$ & 70 & 62 & 61 & 61 & 64 & 52 & 68 & 52 & 63 \\
$0-3$ & 90 & 87 & 84 & 85 & 87 & 83 & 88 & 82 & 85 \\
$0-5$ & 97 & 96 & 94 & 96 & 95 & 94 & 96 & 95 & 95 & 96 \\
\hline
\end{tabular}

Integrated variance is normalized by the total variance in $0 \leq k \leq 30$ and shown in percentages rounded to the nearest integer. Values are shown for ERA-20C (denoted E) and for SPEEDY (denoted S) 
(a) MAM variance

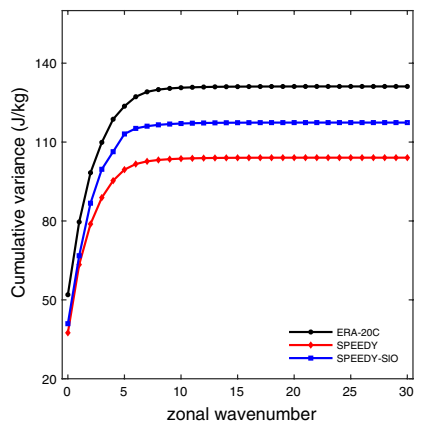

(b) JJA variance

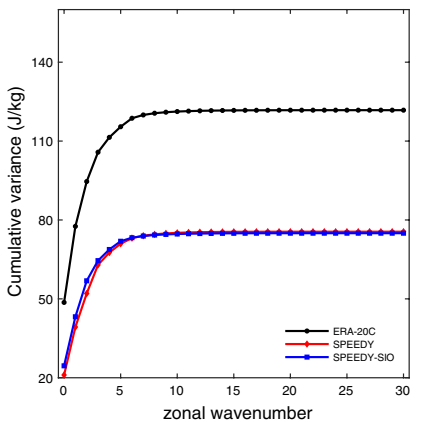

(c) SON variance

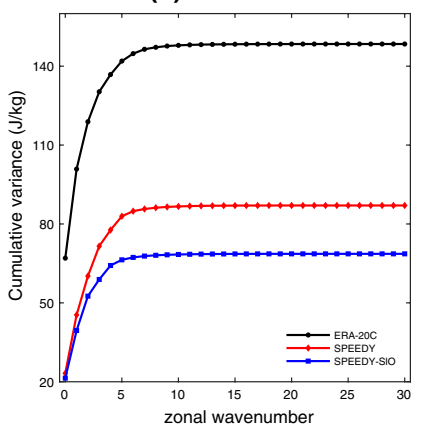

(d) DJF variance

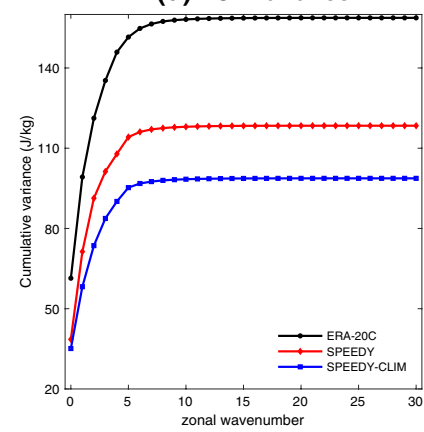

Fig. 7 As in Fig. 6a but for seasonal means. Cumulative variance in a MAM, b JJA, c SON and d DJF seasons

of spatial variability in subsynoptic scales thus extends to temporal domain. Full presentation of the two-dimensional variability distribution are beyond the scope of this paper which uses only a limited amount of the capabilities of the modal decomposition and does not present meridional and vertical scales and Rossby and inertio-gravity modes.

\subsection{Scale-dependent biases}

Now we attempt to discuss variability shortcomings in the model in relation to biases. Similarly to variability, bias is computed by season ( $N=150$, number of months in 50 seasons) and for the annual means (i.e. averaging for the whole dataset, $N=600$, number of months in 50 years) using Eq. (25). The spectrum of bias is compared with the variance spectrum by comparing $V_{k}$ with $B_{k}$ computed as

$B_{k}=\left(2-\delta_{k 0}\right) \sum_{n=1}^{R} \sum_{m=1}^{M} B_{n}^{k}(m)$,

with individual mode bias variance $B_{n}^{k}(m)$ obtained from (26) and $\delta_{k 0}$ defined as in (41).

Biases in SPEEDY and SPEEDY-S1O are computed by comparing them with ERA-20C. Their comparison describes how the slab ocean model changes the scale distribution of atmospheric biases with respect to the SST-forced model. The third bias is one computed as the systematic difference between SPEEDY and SPEEDY-SIO and it represents the perfect-model scenario; assuming a perfect atmosphere model, it assesses the impact of having accurate SSTs. The bias in SPEEDY-S1O against SPEEDY is expected to be smaller than against ERA-20C and this can be checked in Figs. 8a-d and 9.

First, Fig. 8 compares the zonal wavenumber spectra of the bias variance $B_{k}$ computed by Eq. (42) with the variance $V_{k}$, computed by Eq. (41). The main property of the bias variance distribution is its strong scale dependence, similar to the variance, but with a stronger reduction with the zonal scale. The zonal mean state $(k=0)$ is not shown in Fig. 8; the bias in $k=0\left(B_{0}\right)$ largely exceeds the sum of biases in $k>0\left(B_{k>0}\right)$, especially in SPEEDY-S1O. For example, $B_{0}$ is 2-4.2 times, and 1.8-3.4 times greater than $B_{k>0}$ in SPEEDY and SPEEDY-SIO, respectively. The exact numbers depend on season. In SPEEDY-S1O against SPEEDY, $B_{0}$ is at $70 \%$ and $200 \%$ level of that in SPEEDY against ERA-20C in MAM and SON, respectively.

Bias variance exceeds circulation variance in all seasons at nearly all scales. There are however large difference between the seasons. If we use variability in ERA-20C as the reference, then the MAM bias in SPEEDY is smaller than variability at $k=5-6$, baroclinically most active scales. In JJA and SON, biases exceed ERA-20C variability except at $k=6$ in SPEEDY-SIO against SPEEDY in JJA. Furthermore, although large-scale SON bias in SPEEDY-S1O against SPEEDY is smaller than in SPEEDY-S1O against ERA-20C, it is still greater than the bias in SPEEDY against ERA-20C at the same large scales suggesting that large biases introduced by a poor ocean could not be compensated by the perfect atmospheric model. In DJF, biases remain greater than ERA-20C variability except for $k>9$ in the case of SPEEDY-SIO against SPEEDY.

A much larger bias in the zonal mean state in SPEEDYSIO than in SPEEDY is a consequence of unrealistically poor SST produced by the slab-ocean model. As seen in Fig. 1, a poor ocean model increased mean precipitation in addition to making the circulation more zonal (Fig. 4) and inducing larger differences in seasonal variability with respect to reanalysis (Fig. 7). Biases in large-scale waves have also mainly increased, but beyond $k=15$ there is no significant difference between SPEEDY and SPEEDY-SIO (Fig. 8a-d). The same applies to the variance (Fig. 8e-h).

A thorough analysis of biases in different modes would employ Eq. (35) which is outside the scope of the present paper that develops a novel method and demonstrates its power using a relatively simple model with large biases. We limit the present analysis to the comparison of the first two terms on the right-hand side of (35), missing variance in the model and bias variance. Figure 9 visualizes the bias 
(a) MAM, Bias variance

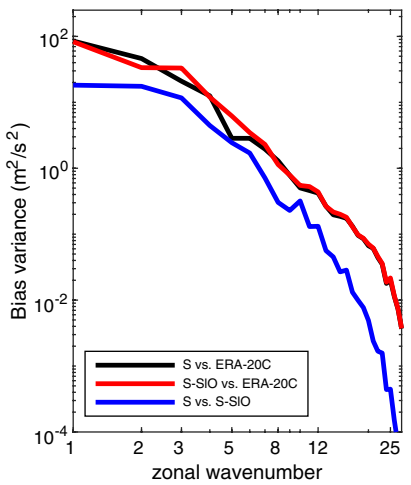

(e) MAM, Variance

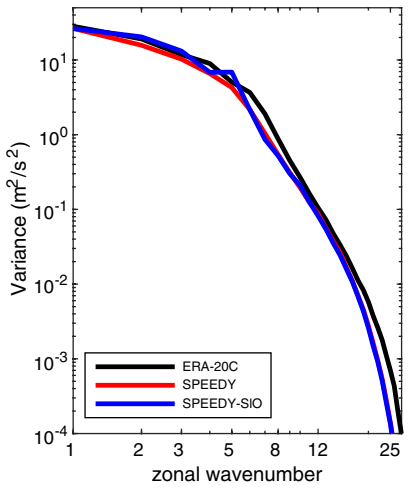

(b) JJA, Bias variance

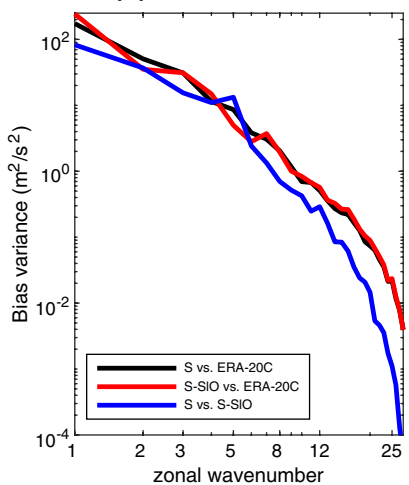

(f) JJA, Variance

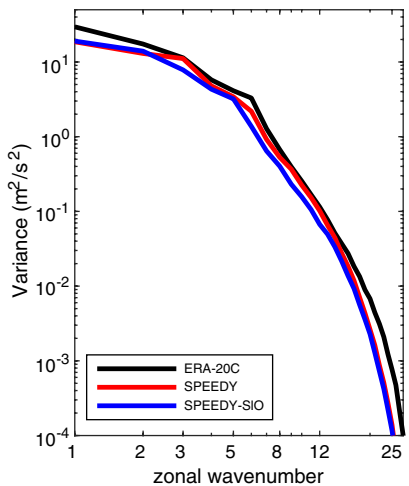

Fig. 8 Seasonal a-d bias variance $B_{k}$ computed by Eq. (42), and $\mathbf{e}-\mathbf{h}$ interannual variance, $V_{k}$, computed by Eq. (41). Bias in a SPEEDY and b SPEEDY-SIO is evaluated with ERA-20C and in c SPEEDY$\mathrm{S} 1 \mathrm{O}$ is evaluated against SPEEDY. The zonal mean state, $k=0$, is not

(c) SON, Bias variance

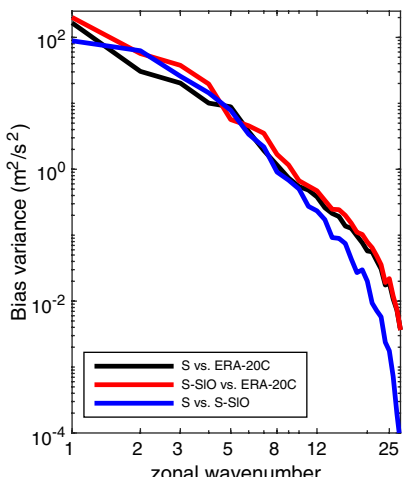

(g) SON, Variance

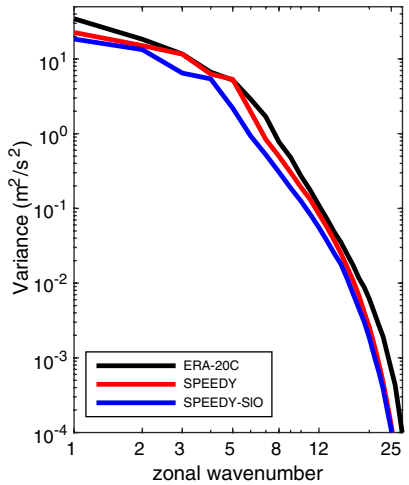

(d) DJF, Bias variance

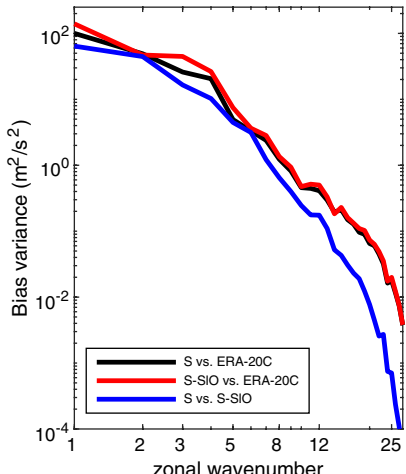

(h) DJF, Variance

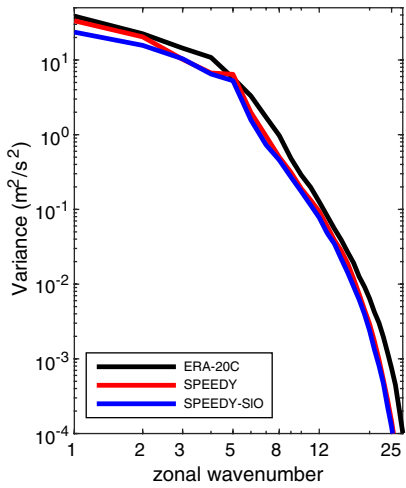

shown. In a-d, the biases of SPEEDY and SPEEDY-SIO relative to ERA-20C are shown in black and red, and the bias of SPEEDY-SIO relative to SPEEDY is shown in blue

(a) SPEEDY vs. ERA-20C

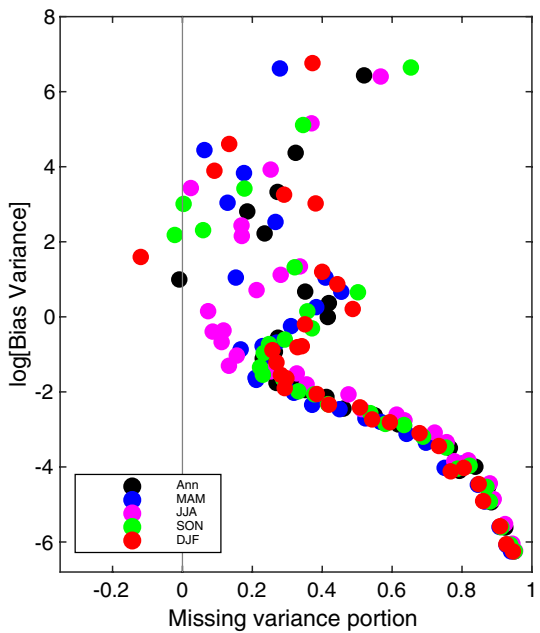

(b) SPEEDY-SIO vs. ERA-20C

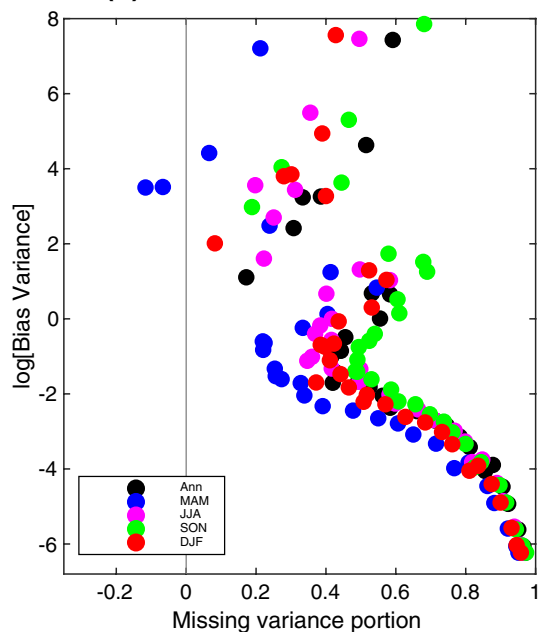

(c) SPEEDY-SIO vs. SPEEDY

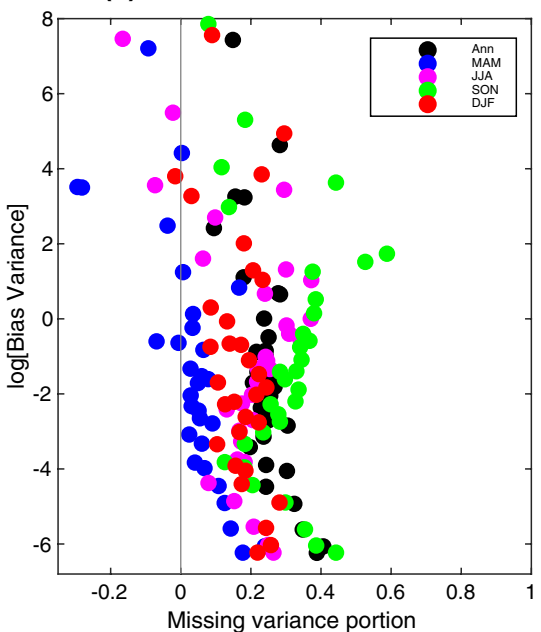

Fig. 9 Scatterplot of the $\operatorname{logarithm}$ of the bias variance, $\log B_{k}$ versus the normalized missing model variance, computed as $\left(V_{k}^{a}-V_{k}^{c}\right) / V_{k}^{a}$, in annual and seasonal means. a SPEEDY and b SPEEDY-SIO evaluated against ERA-20C and c SPEEDY-S1O evaluated against
SPEEDY. Different dots correspond to different zonal wavenumbers $k$ from $k=0$ (top dots with the largest bias variance) to $k=30$ (bottom dots with the smallest bias variance). See text for details 
variance in all $k$ using the logarithmic scale against the relative missing variance which is computed as a difference between the ERA-20C and simulated variance normalized by ERA-20C (or a difference between SPEEDY and SPEEDY-SIO normalized by SPEEDY). Therefore, values smaller than 0 in Fig. 9 imply the variance overestimation whereas values between 0 and 1 denoted increasingly missing variance.

Biases in $k=0$ are represented by the dots at the top of the three panels in Fig. 9. Dots towards the bottom of each panel correspond to successively larger $k$ suggesting an increasing lack of relative variability in the model as the spatial scale reduces (Fig. 9a, b). Biases introduced by the slab-ocean model increase spread in missing portions of variance at the synoptic scales among the seasons that is seen in the area with $\log (B) \approx-2$ (Fig. 9b vs. 9a). On the other hand, Fig. 9c suggests that the missing portion of variance is greatly reduced in the perfect model scenario although large biases remain at planetary scales due to a very poor SST. The large reduction in missing variance at subsynoptic scales is the most noticeable feature in Fig. 9c in support to high-resolution climate modelling providing variability information regardless of the amplitude of large-scale biases.

A two-dimensional analysis of bias in terms of the zonal wavenumbers and meridional modes reveals that the $k=1$ bias in SPEEDY is maximal for $n=1$ meridional mode (not shown). The same $n$ is repeated in SPEEDY-SIO along with another maximum in $n=3$ suggesting an increased wave biases in the midlatitudes. A detailed analysis of the meridional structure and the inertio-gravity component associated with tropical circulation features is left for the subsequent study.

\subsection{Annual cycle of energy distribution}

As suggested by Eq. (35), missing variance and biases are related to deficiencies in mean energy and covariance between the bias and mean state of reanalysis. A detailed evaluation of this relationship is left for a follow-on study using a more realistic climate model. Here, we just illustrates differences in energy distribution among seasons in light of seasonal variations of variability and bias.

The annual cycle of energy distribution is shown in Fig. 10 for two quantities. The first three panels (Fig. 10a-c) show deviations of the climatological monthly energy from the climatological annual mean normalized by the annual mean in the same wavenumber. The climatological annual energy cycle is displayed in other three panels for several wavenumbers (Fig. 10d-f). Figure 10 reveals that shorter scales in the model are too active with respect to their annual mean. For example, wavenumbers $k=5$ and $k=10$ vary up to around $50 \%$ of their annual mean value (Fig. 10b, c) (a) ERA-20C

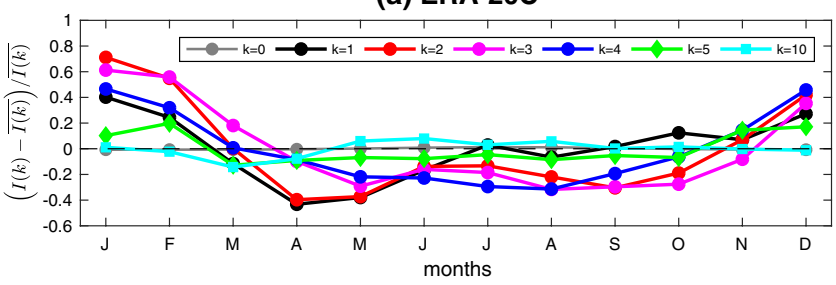

(b) SPEEDY

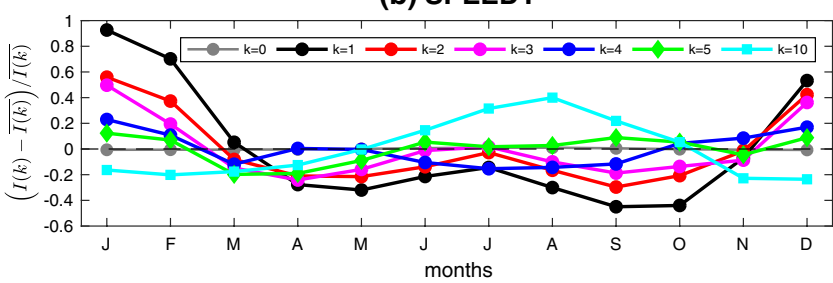

(c) SPEEDY-SIO

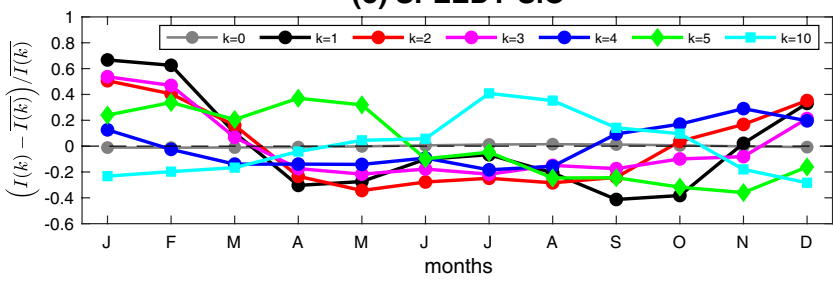

(d) $k=1$

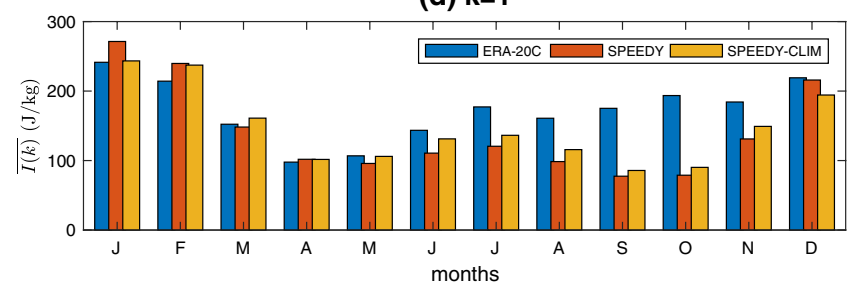

(e) $k=7$

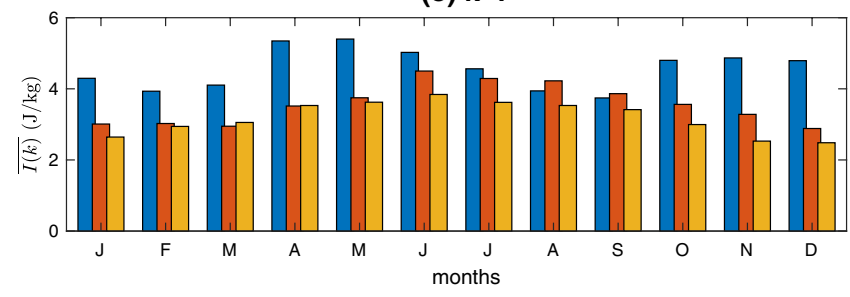

(f) $k=15$

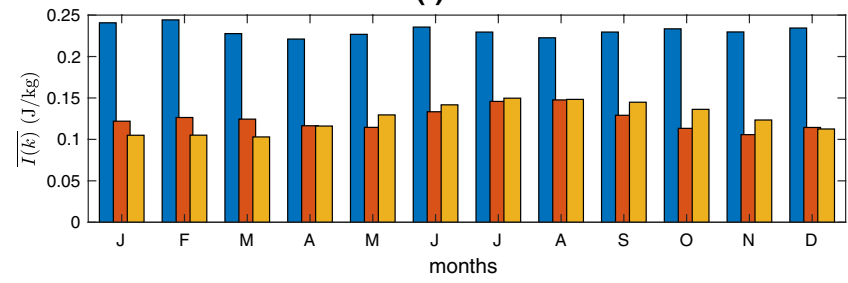

Fig. 10 Deviations of climatological monthly energy from the mean annual energy in selected zonal wavenumbers for a ERA-20C, b SPEEDY and $\mathbf{c}$ SPEEDY-SIO. Climatological annual energy cycle in wavenumbers $\mathbf{d} k=1$, $\mathbf{e} k=7$ and $\mathbf{f} k=15$ 
that is not seen in ERA-20C. The same behaviour is found also for other $k<20$, especially in JJA in SPEEDY-SIO. The wavenumber $k=1$, which should have a positive deviation $10-20 \%$ in SON according to ERA-20C, is around $40 \%$ under-active in the model with respect to the annual average. At the same time, the underestimation of $k=1$ energy deviation in SON may be less apparent in the model outputs because its amplitude is less than half that of the reanalysis (Fig. 10d). We may recall here that the model is characterized by large biases in $k=1$ in the same season (Figs. 8, 9). As the scale reduces, the annual cycle nearly disappears in ERA-20C as illustrated by $k=15$ in Fig. 10a, f. In the model however, the annual cycle of variability at these scales is still significant (Fig. 10b, c) even though its amplitude is small (Fig. 10f).

\section{Biases in physical space}

The presented modal decomposition of bias and variability calls for further investigation using state-of-the-art climate models. However, the modal decomposition alone can not tell us what exactly to change in the model. It can guide the physical space analysis and sensitivity studies that should lead to improved models. In the present case, large biases have been expected as SPEEDY was designed and tuned with the aim of simulating mid-latitude $\mathrm{NH}$ tropospheric variability. In particular, SPEEDY stratosphere should be regarded as an upper boundary condition as the top layer has a strong damping term ("sponge layer"), to avoid strong reflection of waves. However, damping of planetary waves at the top of a model has the undesired side effect on stratosphere motions.

This was evidenced in Fig. 2i-1 showing biases in the zonal mean wind $(k=0)$ in SPEEDY with a large negative bias under the tropical tropopause and a large positive bias in the subtropical stratosphere. The largest bias in the upper troposphere wind is found in DJF season between $50^{\circ} \mathrm{S}$ and $60^{\circ} \mathrm{S}$ throughout most of the troposphere (Fig. 21). The horizontal structure of biases in Fig. 11 is shown at the model level 2 which is close to $100 \mathrm{hPa}$ and under the influence of a strong damping at the model top level above. There is a negative zonal wind bias in the tropics and a positive bias in the midlatitudes. Therefore, $\mathrm{SH}$ westerlies are too strong in SPEEDY as mentioned in Sect. 3.2.

Although biases in Fig. 11 can be computed directly in physical space, they can also be calculated for physical space by using $\overline{\Delta \chi_{n}^{k}(m)}$ in Eq. (16) followed by Eq. (15). Similarly, bias in physical space for any $k>0$ or a combination of $(k, n, m)$ indices is obtained by setting $\overline{\Delta \chi_{n}^{k}(m)}=0$ for all other $k, n$ and $m$ indices and solving Eqs. $(15,16)$. Such modal filtering relies on the idea that normal-mode functions, although not the normal modes of the model, represent physically meaningful spatial patterns, especially in comparison to statistical analysis such as EOFs. A number of previous studies successfully employed this assumption to investigate different aspects of dynamics (e.g. Kasahara and Tanaka 1989; Tanaka and Kung 1989; Castanheira et al. 2002; Blaauw and Žagar 2018). Figures 12 and 13 filter only zonal scales, i.e. the meridional and vertical structure is due to the completeness of $(15,16)$ retained.

Figure 12 shows wind biases in $k=1$ and $k=2$ in DJF. It suggests that $k=1$ bias in the tropics are coupled to the extra-tropical biases in NH winter (Fig. 12a). Bias in the meridional wind over the maritime continent has the same sign in $k=1$ and $k=2$ and produces the negative maximum of the total $v$ bias that is most likely responsible for the model too strong convection and precipitation (Fig. 1) over the region. This process through teleconnections may cause negative zonal wind biases over Northern Pacific and positive biases over Mediterranean in Fig. 12a.

Figure 12 also suggests that the absolute magnitude of the large-scale bias is largest in the subtropics. The same region is characterized by largest underestimation of interannual variability in physical space as discussed in Sect. 3.2. The vertical structure along a single latitude circle in the subtropics Fig. 13 (a) DJF, $u$ wind, lev 2

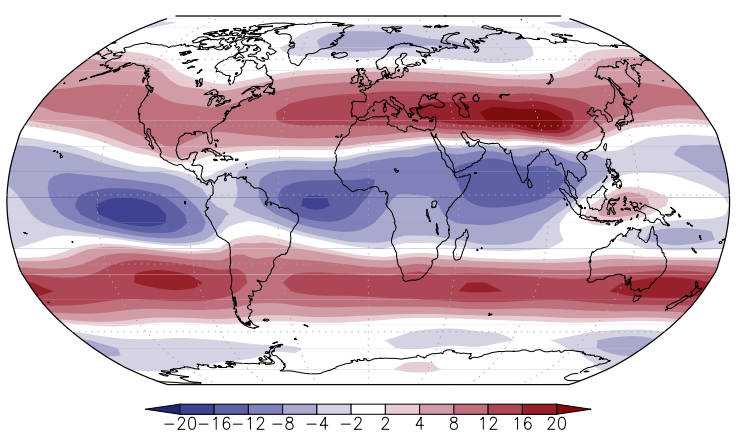

(b) DJF, v wind, lev 2

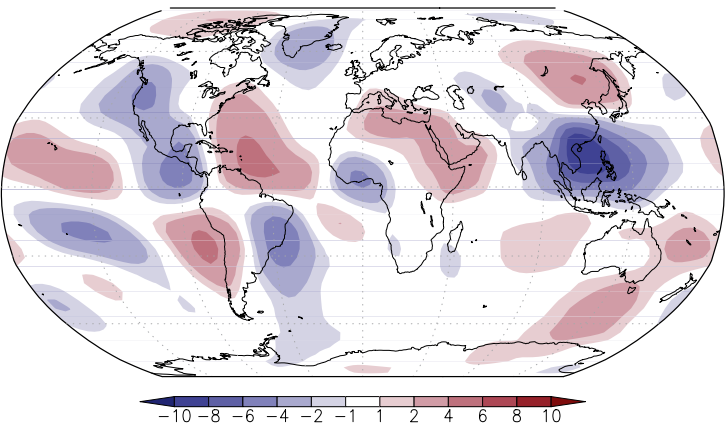

Fig. 11 Wind biases in SPEEDY in DJF in physical space. a Zonal and $\mathbf{b}$ meridional wind biases at level $2(\sim 100 \mathrm{hPa})$. Units are $\mathrm{m} / \mathrm{s}$ 
(a) $u$ wind, lev 2, DJF, $k=1$

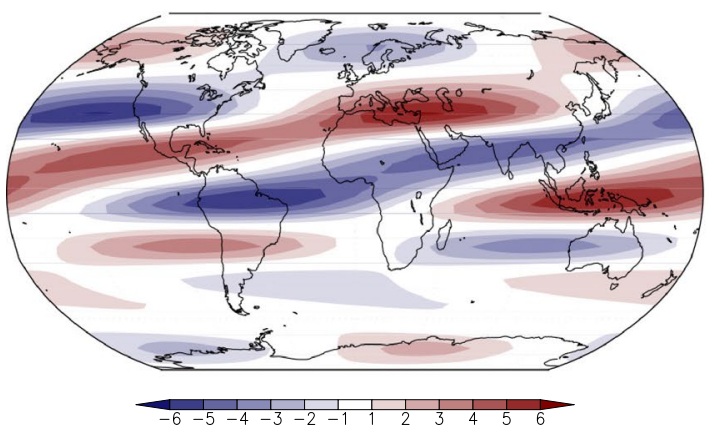

(c) $\vee$ wind, lev $2, D J F, k=1$

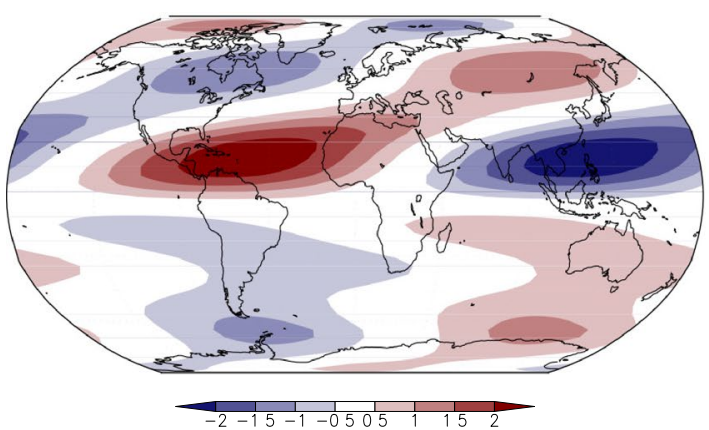

(b) $u$ wind, lev $2, \mathrm{DJF}, \mathrm{k}=2$

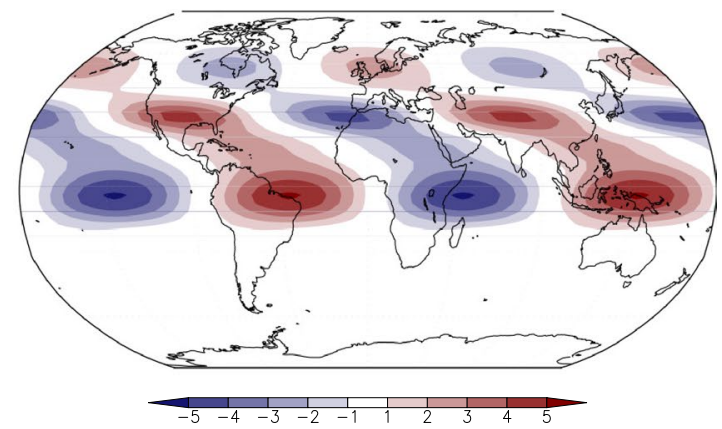

(d) $\vee$ wind, lev $2, D J F, k=2$

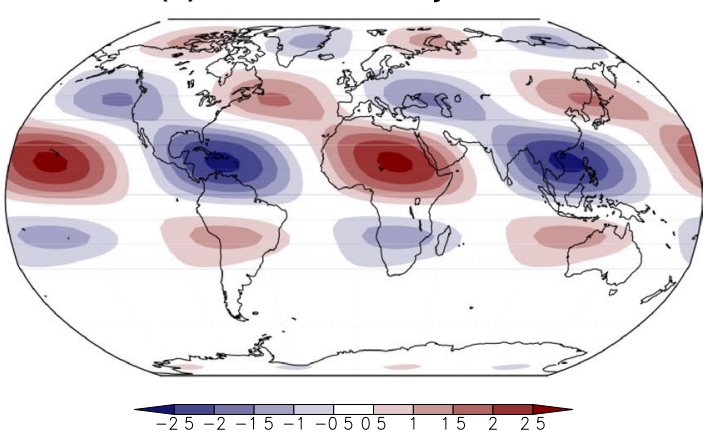

Fig. 12 DJF biases at model level 2 in $\mathbf{a}, \mathbf{b}$ zonal wind and $\mathbf{b}, \mathbf{c}$ meridional wind for zonal wavenumbers $\mathbf{a}, \mathbf{c} k=1$ and $\mathbf{b}, \mathbf{d} k=2$. Units are $\mathrm{m} / \mathrm{s}$

(a) $u$ wind, DJF, $k=1$

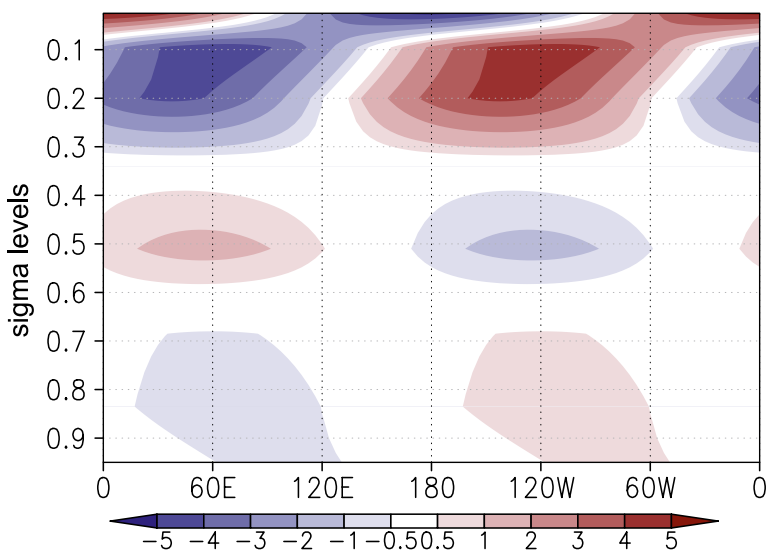

(b) T, DJF, k=1

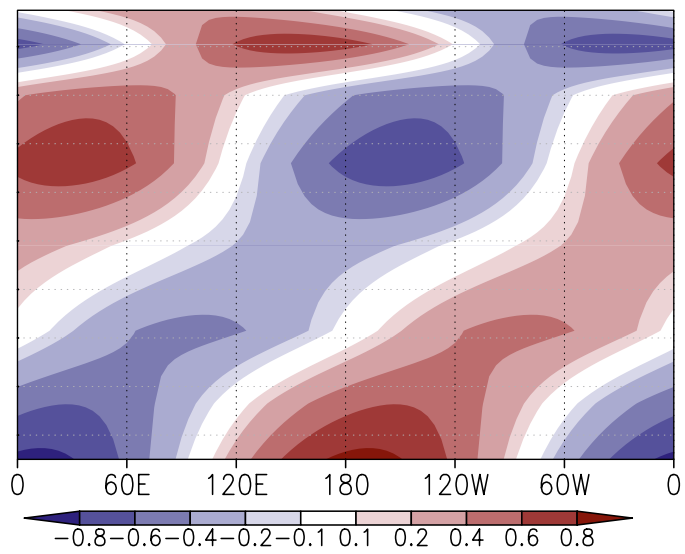

Fig. 13 Longitudinal structure of the bias in $k=1$ along latitude circle $16.7^{\circ} \mathrm{N}$ in DJF in a zonal wind (in $\mathrm{m} / \mathrm{s}$ ) and $\mathbf{b}$ temperature (in $\mathrm{K}$ )

shows the baroclinic vertical structure of the zonal wind bias and the coupling between the surface temperature bias in the Atlantic and western Pacific with the upper troposphere biases in temperature in the opposite hemisphere. It confirms the horizontal and vertical couplings between biases in remote regions, as discussed by Wang et al. (2014).

\section{Conclusions and outlook}

Spatio-temporal variability and biases in climate model thermodynamical fields and circulation are coupled, calling for an approach that considers them simultaneously. 
That was achieved in this paper by using the normal-mode function decomposition of the GCM outputs and reanalysis data to assess variability and bias as a function of the zonal wavenumber and the meridional and vertical modes. An analytical relationship has been derived that describes, for each mode, deficiencies in simulated mean circulation and temporal variance as a function of the bias amplitude and the covariance between the reanalysis mean state and bias.

The method was demonstrated using SPEEDY, a primitive-equation GCM with 8 terrain-following vertical levels (near pressures 950, 835, 685, 510, 340, 200, 95 and $25 \mathrm{hPa}$ ) and horizontal resolution T30. The model was compared with the ERA-20C reanalyses at the same horizontal and vertical grid. Unlike standard reanalyses that use all available observations, ERA-20C is based on surface observations in 20th century. The spatio-temporal variances in ERA-20C and SPEEDY were computed for the period 1961-2010.

It was found that about $48 \%$ of the interannual variance in ERA-20C, weighted across all meridians and pressure levels, is associated with the zonal mean state (zonal wavenumber $k=0$ ). The smaller the scale, the less variance so that $80-90 \%$ (depending on season) of the variance is at $k$ $=0-3$ whereas in scales smaller than zonal wavenumber $k=7$ there is only about $1 \%$ of the total interannual variance. The season with the largest interannual variance is boreal winter, while JJA has on average about 30\% less variance than DJF season. Similarly, JJA is characterized by about one-third lower percentage of wave $(k>0)$ energy in total $(k \geq 0)$ energy.

These properties of the ERA-20C data were used to validate SPEEDY. In one setup, the model was used with the prescribed SST from ERA-20C (SPEEDY run) whereas another simulation applied a slab ocean model which updates SST in each forecast step (SPEEDY-SIO run). A relatively good representation of the average precipitation, winds and temperature in SPEEDY became worse in SPEEDY-SIO. Scale validation revealed that the model underestimates interannual variance at all scales but especially at large scales, and that the variance underestimation in SPEEDY-S1O oscillates among the seasons compared to the SST-forced run. The comparison of energy spectra also shows a poor representation of the annual energy cycle at planetary scales. The missing temporal variance in the model relative to ERA-20C becomes larger for smaller scales. This is due an overall lack of small scale variability in SPEEDY, expected in part since ERA-20C was produced at a higher resolution and truncated to T30.

Similar to variability, bias is strongly scale dependent; the larger the scale, the greater the bias. Biases present in SPEEDY increase in SPEEDY-S1O, especially in the zonal mean state throughout the model atmosphere. The comparison of bias in a perfect-model scenario, defined as a systematic difference between the SPEEDY-SIO and SPEEDY experiments, suggests that improving the atmospheric model can greatly increase the variance in the model on synoptic and subsynoptic scales. However, large biases associated with a poor SST remain at planetary scales.

We have not discussed relative amplitudes of various terms of the Eq. (35) describing relationship between energy and variance in the model and reanalysis, bias variance and covariance between the reanalysis mean state and bias. This is left for follow-on studies with a more realistic model. Much of the bias in the present study is in $k=0$ because the applied model does not have an active stratosphere. It is nevertheless demonstrated how biases adjust to changes in simulated variance across scales. For example, the season with the smallest variability (SON) in the coupled model is characterised by biases that have amplitudes greater than ERA-20C variability, regardless of the perfect-model scenario.

The modal method enables scale-selected filtering of the bias to physical space. Presented depiction of biases in zonal wavenumbers $k=1$ and $k=2$ demonstrates that the largescale biases have a meridionally and vertically tilted structure suggesting a coupling between biases in remote regions. In the present experiments, temperature biases near the surface in the Atlantic and western Pacific appear coupled with the upper-troposphere biases of the same sign far eastward.

Follow-on studies will provide a discussion of the twodimensional distribution of variability and bias associated with the quasi-geostrophic (or Rossby modes) and unbalanced dynamics (or inertio-gravity modes) using a more advanced GCM. The modal decomposition is especially useful in the tropics where other global approaches (e.g. spherical harmonics) do not filter the equatorial wave properties. For the validation and climate model intercomparison, a limited number of modes, considerably smaller than the actual number of degrees of freedom of a climate model, may suffice for the multi-model analysis as a majority of variability and bias is at large scales. The first application of the method to daily data for the computation of subseasonal variability spectra and trends in the ERA-Interim reanalysis has already been performed (Žagar et al. 2018).

Acknowledgements The authors are grateful to the comments of two anonymous reviewers that led to the paper improvements. Discussions with Grant Branstator, Akira Kasahara, Fred Kucharski, Franco Molteni and Joe Tribbia are gratefully acknowledged. Nedjeljka Žagar and Martin Horvat were partly supported by the Slovenian Research Agency, program P1-0188 and project J1-9431. Funded by the European Research Council, Grant Agreement no. 280153.

\section{References}

Blaauw M, Žagar N (2018) Multivariate analysis of Kelvin wave seasonal variability in ECMWF L91 analyses. Atmos Chem Phys 18:8313-8330. https://doi.org/10.5194/acp-18-8313-2018 
Blažica V, Žagar N, Strajnar B, Cedilnik J (2013) Rotational and divergent kinetic energy in the mesoscale model ALADIN. Tellus $65 \mathrm{~A}(18): 918$

Branstator G (2002) Circumglobal teleconnections, the jet stream waveguide, and the north atlantic oscillation. J Clim 15:1893-1910

Castanheira JM (2000) Climatic variability of the atmospheric circulation at the global scale. PhD thesis, Department of Physics, University of Aveiro, Portugal

Castanheira JM, Graf HF, DaCamara C, Rocha A (2002) Using a physical reference frame to study global circulation variability. J Atmos Sci 59:1490-1501

Charlton-Perez AJ, Baldwin MP, Birner T, Black RX, Butler AH, Calvo N, Davis NA, Gerber EP, Gillett N, Hardiman S, Kim J, Krüger K, Lee Y, Manzini E, McDaniel BA, Polvani L, Reichler T, Shaw TA, Sigmond M, Son S, Toohey M, Wilcox L, Yoden S, Christiansen B, Lott F, Shindell D, Yukimoto S, Watanabe S (2013) On the lack of stratospheric dynamical variability in low-top versions of the cmip5 models. J Geophys Res Atmos 118:2494-2505

Cubasch U, Wuebbles D, Chen D, Facchini M, Frame D, Mahowald $\mathrm{N}$, Winther JG (2013) Introduction. Chapter 1 in climate change 2013: the physical science basis. In: Stocker TF, Qin D, Plattner G-K, Tignor M, Allen SK, Boschung J, Nauels A, Xia Y, Bex V, Midgley PM (eds) Contribution of working group I to the fifth assessment report of the intergovernmental panel on climate change. Cambridge University Press, Cambridge. https://doi. org/10.1017/CBO9781107415324.007

Daley R (1991) Atmospheric data analysis. Cambridge University Press, Cambridge, p 460

Ehret U, Zehe E, Wulfmeyer V, Warrach-Sagi K, Liebert J (2012) Should we apply bias correction to global and regional climate model data? Hydrol Earth Syst Sci 16:3391-3404

Eyring V, Bony S, Meehl GA, Senior C, Stevens B, Stouffer R, Taylor K (2016) Overview of the coupled model intercomparison project phase 6 (CMIP6) experimental design and organization. Geosci Model Dev 9:1937-1958

Feudale L, Kucharski F (2013) A common mode of variability of African and Indian monsoon rainfall at decadal timescale. Clim Dyn 41:243-254

Flato G, Marotzke J, Abiodun B, Braconnot P, Chou S, Collins W, Cox P, Driouech F, Emori S, Eyring V, Forest C, Gleckler P, Guilyardi E, Jakob C, Kattsov V, Reason C, Rummukainen M (2013) Evaluation of climate models. Chapter 9 in climate change 2013: the physical science basis. In: Stocker TF, Qin D, Plattner G-K, Tignor M, Allen SK, Boschung J, Nauels A, Xia Y, Bex V, Midgley PM (eds) Contribution of working group I to the fifth assessment report of the intergovernmental panel on climate change. Cambridge University Press, Cambridge. https ://doi.org/10.1017/CBO9781107415324.020

Held I, Suarez M (1994) A proposal for the intercomparison of dynamical cores of atmospheric general circulation models. BAMS 75:1825-1830

Herceg-Bulić I, Kucharski F (2012) Delayed ENSO impact on spring precipitation over North/Atlantic European region. J Atmos Sci 51:2225-2237

Herceg-Bulić I, Kucharski F (2013) North Atlantic SSTs as a link between the Wintertime NAO and the following spring climate. J Clim 27:186-201

Hoskins BJ, James IN (2014) Fluid dynamics of the midlatitude atmosphere. Wiley, Oxford

Hough SS (1898) On the application of harmonic analysis to the dynamical theory of the tides-Part II. On the general integration of Laplaceś dynamical equations. Philos Trans R Soc Lond A191:139-185
Hung MP, Lin JL, Wang W, Kim D, Shinoda T, Weaver SJ (2013) MJO and convectively coupled equatorial waves simulated by CMIP5 climate models. J Clim 26:6185-6214

Kasahara A (1976) Normal modes of ultralong waves in the atmosphere. Mon Weather Rev 104:669-690

Kasahara A (1980) Effect of zonal flows on the free oscillations of a barotropic atmosphere. J Atmos Sci 37:917-929 (Corrigendum, J. Atmos. Sci., 38 (1981), 2284-2285)

Kasahara A, Puri K (1981) Spectral representation of three-dimensional global data by expansion in normal mode functions. Mon Weather Rev 109:37-51

Kasahara A, Tanaka H (1989) Application of vertical normal mode expansion to problems of baroclinic instability. J Atmos Sci 46:489-510

Knutti R, Sedlacek J (2013) Robustness and uncertainties in the new CMIP5 climate model projections. Nat Clim Change 3:369-373

Kucharski F, Molteni F, Bracco A (2006) Decadal interactions between the western tropical Pacific and the North Atlantic Oscillation. Clim Dyn 26:79-91

Lin JL, Kiladis G, Mapes BE, Weickmann KM, Sperber KR, Lin W, Wheeler MC, Schubert SD, Genio AD, Donner LJ, Emori S, Gueremy JF, Hourdin F, Rasch PJ, Roeckner E, Scinocca JF (2006) Tropical intraseasonal variability in 14 IPCC AR4 climate models. Part I: convective signals. J Clim 19:2665-2690

Machenhauer B (1977) On the dynamics of gravity oscillations in a shallow water model, with applications to normal mode initialization. Beitr Phys Atmos 50:253-271

Marques CAF, Castanheira JM (2012) A detailed normal-mode energetics of the general circulation of the atmosphere. J Atmos Sci 69:2718-2732

Molteni F (2003) Atmospheric simulations using a GCM with simplified physical parametrizations. I: model climatology and variability in multi-decadal experiments. Clim Dyn 20:175-195

Navarra A, Stern W, Miyakoda K (1994) Reduction of the Gibbs oscillation in spectral model simulations. J Clim 7:1169-1183

Palmer T, Doblas-Reyes F, Weisheimer A, Rodwell M (2008) Toward seamless prediction: calibration of climate change projections using seasonal forecasts. Bull Am Meteorol Soc 89:459-470

Phillips NA (1990) Dispersion processes in large-scale weather prediction. Sixth IMO lecture, World Meteorological Organization, No, p 700

Poli P, Hersbach H, Dee P, Berrisford P, Simmons A, Vitart F, Laloyaux P, Tan D, Puebey C, Thépaut J, Trémolet Y, Hólm E, Bonavita M, Isaksen L, Fisher M (2016) ERA-20C: an atmospheric reanalysis of the twentieth century. J Clim 29:4083-4097

Polo I, Martin-Rey M, Rodriguez-Fonesca B, Kucharski F, Mechoso C (2014) Processes in the Pacific La Nina onset triggered by Atlantic Nino. Clim Dyn

Rayner N, Brohan P, Parker D, Folland C, Kennedy J, Vanicek M, Ansell T, Tett S (2006) Improved analyses of changes and uncertainties in sea surface temperature measured in situ since the mid-nineteenth century: the HadSST2 dataset. J Clim 19:446-469

Scaife A, Kucharski F, Folland C, Kinter J, Bronnimann S, Fereday D, Fischer A, Grainger S, Jin E, Kang I, Knight J, Kusunoki S, Lau N, Nath M, Nakaegawa T, Pegion P, Schubert S, Sporyshev P, Syktus J, Yoon J, Zeng N, Zhou T (2009) The CLIVAR C20C project: selected twentieth century climate events. Clim Dyn 33:603-614

Scaife AA, Woollings T, Knight J, Martin G, Hinton T (2012) Atmospheric blocking and mean biases in climate models. J Clim 23:6143-6152

Shepherd TG (2014) Atmospheric circulation as a source of uncertainty in climate change projections. Natu Geosci 7:703. https:// doi.org/10.1038/ngeo2253 
Staniforth A, Beland M, Coté J (1985) An analysis of the vertical structure equation in sigma coordinates. Atmos-Ocean 23:323-358

Sun C, Kucharski F, Li J, Jin F-F, Kang I-S, Ding R (2017) Western tropical Pacific multidecadal variability forced by the Atlantic multidecadal oscillation. Nat Commun 8:15998. https://doi. org/10.1038/ncomms 15998

Swarztrauber PN, Kasahara A (1985) The vector harmonic analysis of laplace tidal equations. SIAM J Stat Comput 6:464-491

Tanaka H (1985) Global energetics analysis by expansion into threedimensional normal-mode functions during the FGGE winter. J Meteorol Soc Jpn 63:180-200

Tanaka H, Kimura K (1996) Normal-mode energetics analysis and the intercomparison for the recent ECMWF, NMC, and JMA global analyses. J Meteorol Soc Jpn 74:525-538

Tanaka H, Kung E (1988) Normal-mode expansion of the general circulation during the FGGE year. J Atmos Sci 45:3723-3736

Tanaka H, Kung E (1989) A study of low-frequency unstable planetary waves in realistic zonal and zonally varing basic states. Tellus 41A:179-199

Terasaki K, Tanaka H (2007) An analysis of the 3-D atmospheric energy spectra and interactions using analytical vertical structure functions and two reanalyses. J Meteorol Soc Jpn 85:785-796

Vitart F, Buizza R, Balmaseda MA, Balsamo G, Bidlot JR, Bonet A, Fuentes M, Hofstadler A, Molteni F, Palmer T (2008) The new VAREPS-monthly forecasting system: a first step towards seamless prediction. Q J R Meteorol Soc 134:1789-1799
Wang C, Zhang L, Lee SK, Wu L, Mechoso CR (2014) A global perspective on CMIP5 climate model biases. Nat Clim Change 4:201-205. https://doi.org/10.1038/nclimate2118

Wedi NP (2014) Increasing horizontal resolution in numerical weather prediction and climate simulations: illusion or panacea? Philos Trans R Soc Lond A Math Phys Eng Sci 372:2018. https://doi. org/10.1098/rsta.2013.0289

Žagar N, Kasahara A, Terasaki K, Tribbia J, Tanaka H (2015) Normalmode function representation of global 3D datasets: open-access software for the atmospheric research community. Geosci Model Dev 8:1169-1195

Žagar N, Jelić D, Blaauw M, Bechtold P (2017) Energy spectra and inertia-gravity waves in global analyses. J Atmos Sci 74:2447-2466

Žagar N, Jelić D, Alexander J, Manzini E (2018) Estimating subseasonal variability and trends in global atmosphere using reanalysis data. Geophy Res Lett 45:12999-13007. https://doi. org/10.1029/2018GL080051

Publisher's Note Springer Nature remains neutral with regard to jurisdictional claims in published maps and institutional affiliations. 